\title{
Optical Disk Scanning Technology
}

\section{Tetsuo Saimi}

Matsushita Electric Industrial Co., Ltd.

Kadoma, Osaka, Japan

\section{CONTENTS}

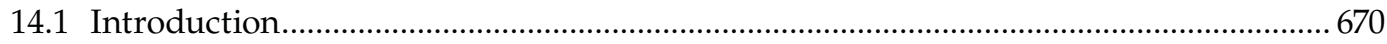

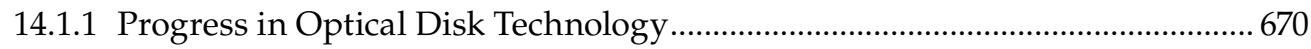

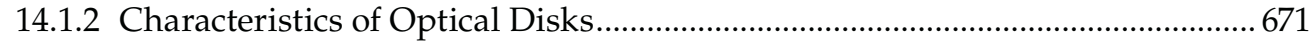

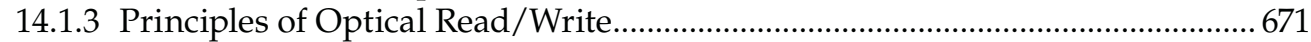

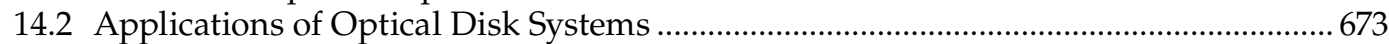

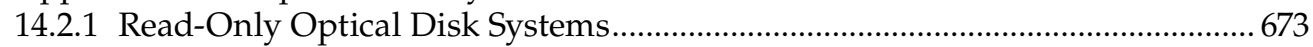

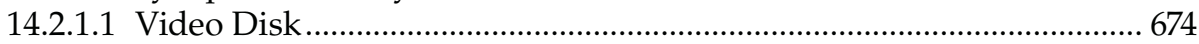

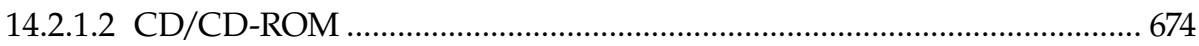

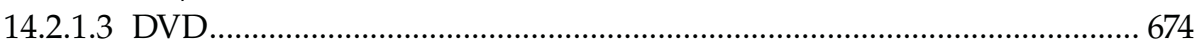

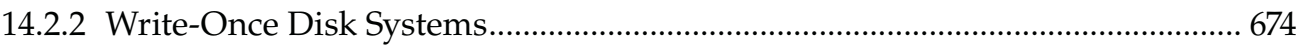

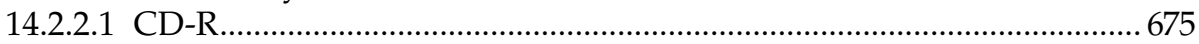

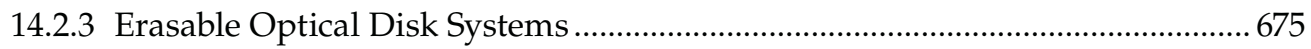

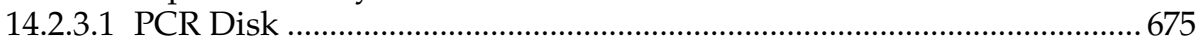

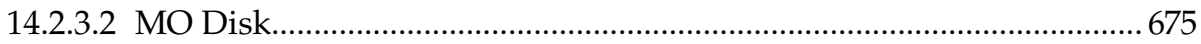

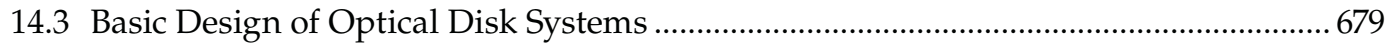

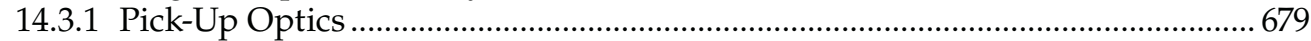

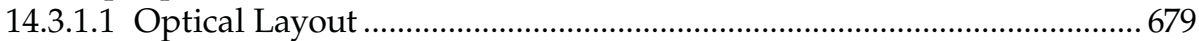

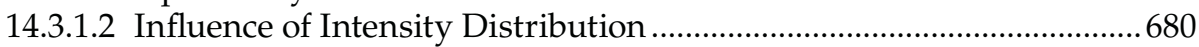

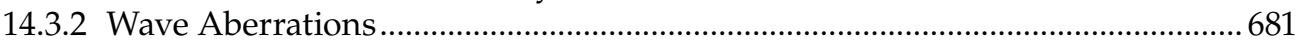

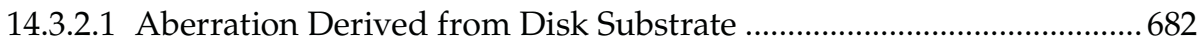

14.3.2.2 Wave Aberrations of Optical Components ..............................................68 683

14.3.2.3 Aberration Due to the Semiconductor Laser ............................................68 683

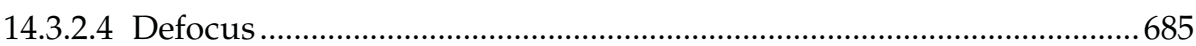

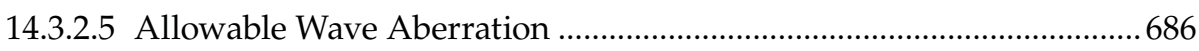

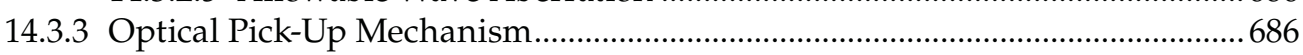

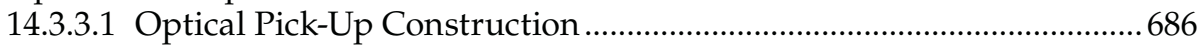

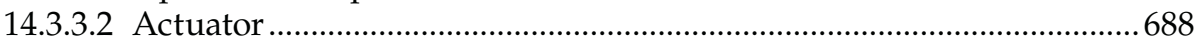

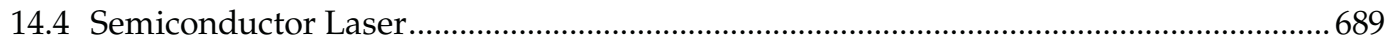

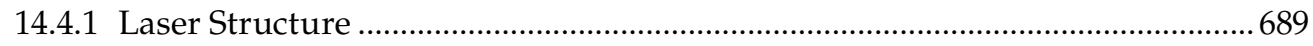

14.4.1.1 Operating Principles of an Al-Ga-As Double Heterojunction

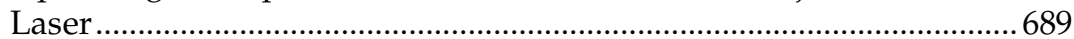

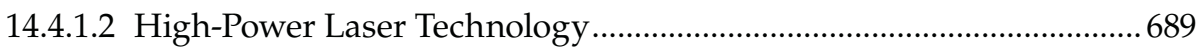

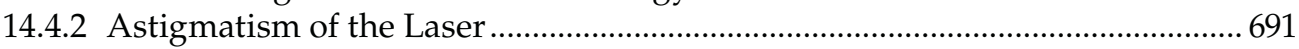

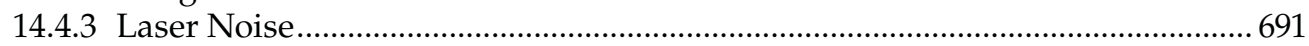

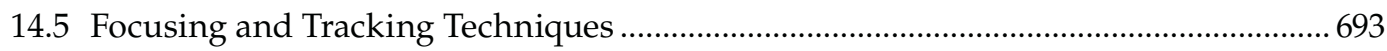

14.5.1 Focusing Servo System and Method of Error Signal Detection .......................... 693 


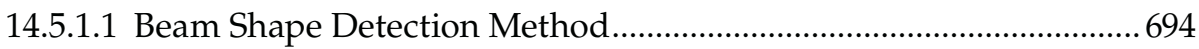

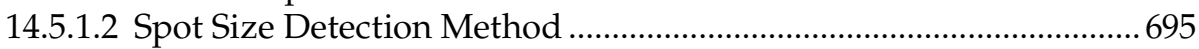

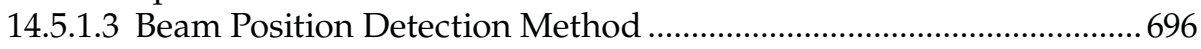

14.5.1.4 Beam Phase Difference Detection .....................................................698

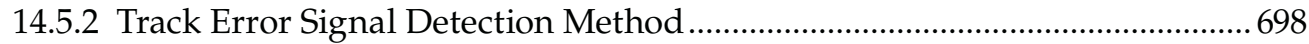

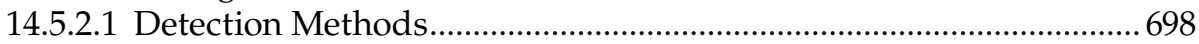

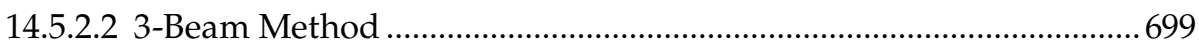

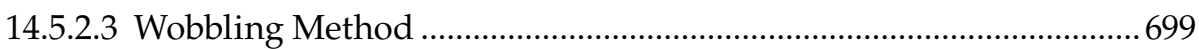

14.5.2.4 Differential Phase Detection (DPD) Method .....................................699

14.5.2.5 Push-Pull Track Error Signal Detection Method ...............................700

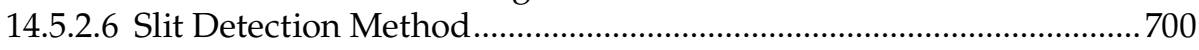

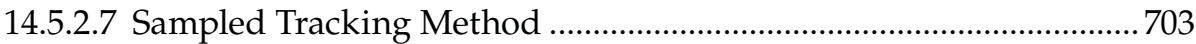

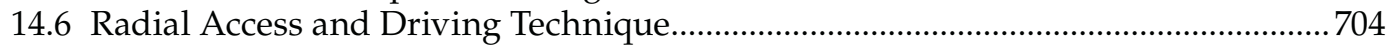

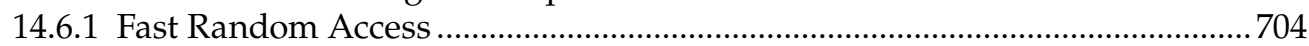

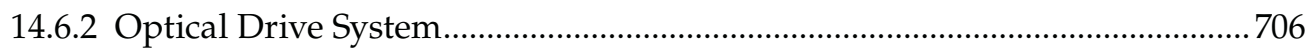

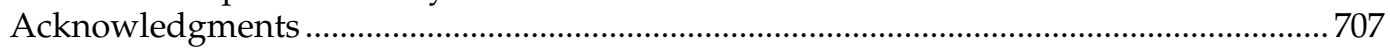

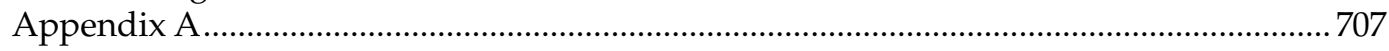

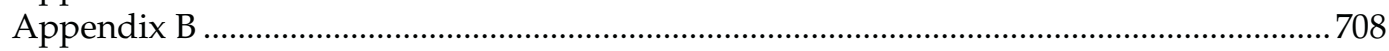

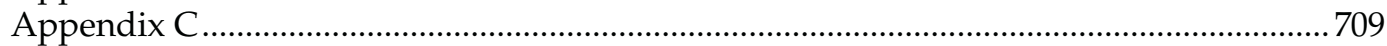

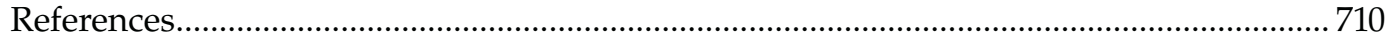

\subsection{INTRODUCTION}

The aim of this chapter is to describe important aspects of optical disk recording and readout technologies, with a brief historical introduction and references for further study. The selected topics are based on the contemporary analysis and experimental results of general interest.

\subsubsection{Progress in Optical Disk Technology}

The fundamental concept of an optical disk dates back to 1961 when Stanford Research Laboratories developed a video disk using photographic technology. However, the low luminance of available light sources yielded reproduced images of low quality. Columbia Broadcasting System (CBS) announced the EVR (Electronic Video Recorder) system in 1967, but enormous costs ultimately forced them to discontinue development. The invention of the laser by T. H. Maiman et al. in 1960 provided the light source considered the most suitable for optical disks.

Lasers have good temporal and spatial coherence, which enables one to obtain the small, diffraction-limited beam spot necessary for high-quality information retrieval from optical disks. After many approaches were considered, the basic design of optical disks, the "bit-by-bit" recording method, was developed in the 1970s. The first optical video disk system for commercial use, the VLP (video long play), was released in 1973 by Philips of Holland and MCA (Music Corporation of America) of the United States. In early systems, the He-Ne laser was the preferred light source. The introduction of many new optical disk systems soon followed. The 12-cm diameter digital audio disk (DAD), later called the CD (compact disk), was announced in 1978. Standardized CD products from several 
manufacturers became available in December 1982. CD players use semiconductor lasers to allow the design of small and lightweight players. In 1996, the digital versatile disk (DVD) for players was released. These playback-only systems marked the inception of optical disk products. Write-once optical disk systems were first introduced by Philips in 1978.

Development of rewritable optical disk systems accelerated in the 1980s as the performance of reversible media progressed. Magneto-optical (MO) disks that utilize a magnetic field reversal for recording and the Kerr effect for playback were commercialized in 1988 by Sony. In 1989, the first phase-change rewritable (PCR) disk, containing 470 megabytes user capacity and utilizing an amorphous-to-crystalline phase change ${ }^{1}$ for recording and playback was commercialized by Matsushita. In 2000, rewritable DVDs (DVD-RAM, -RW) were released and the development for higher density DVD media started.

\subsubsection{Characteristics of Optical Disks}

Optical disks are now used in various applications, including audio, computer memory devices, picture files, document files, and video files. The advantages of optical disks over other known memory devices are:

1. Large capacity/high information density. The information capacity of a $120-\mathrm{mm}$ diameter DVD disk is 4.7 Gbytes for single-layer ROM and RAM, and 8.5 Gbytes for double-layer ROM. The recording density of commercial products is about 3.3 Gbits/in ${ }^{2}$ for DVD-ROM and 4 Gbits/in² for DVD-RAM. Recent developments for next generation products show that an information density of more than 16 Gbits/in ${ }^{2}$ can be achieved by using a blue laser and an objective lens (OB) of high numerical aperture (NA).

2. Fast random-access library systems allow access to large mass memories. Changing mechanisms provide access within seconds to several petabytes of information.

3. Reliability. The information surface of an optical disk is covered with a protective layer, which ensures a long archival life. Information retrieval is achieved without physical contact between the optical pick-up and disk, which increases the reliability of stored information.

4. Replication. Mass production using injection molding or other high-volume techniques is possible. Replicated optical disks benefit from lower cost per bit than the rigid magnetic disk or tapes.

5. Removability/ROM-RAM compatibility. A large quantity of data can be handled easily by exchanging disks. Compatibility between replicated and recordable disks and interchangeability between standardized drives provide this capability. These advantages lead to the ubiquitous uses of optical disk products in consumer and computer applications.

\subsubsection{Principles of Optical Read/Write ${ }^{2-6}$}

In many optical disks, as in the normal audio disk, information is recorded in a spiral groove referred to as the "track." The information cells shown in Figure 14.1 are called "pits," or "marks." They are discontinuous small depressions, differential reflectivity patterns or phase-shifting patterns, all showing differential reflectivity. Information signals (SGs)are derived from changes in luminance caused by diffraction of the laser beam by the pits or marks (which are about $0.3-\mu \mathrm{m}^{2}$ diffraction cells). The laser beam emerging from 
(a)

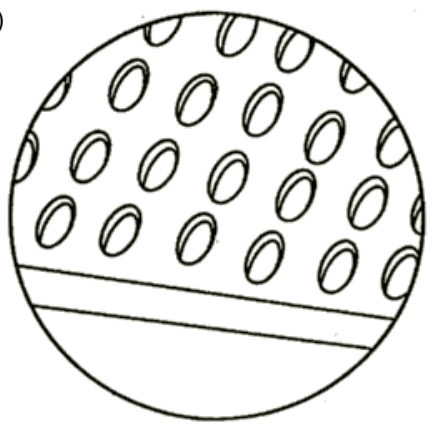

(b)

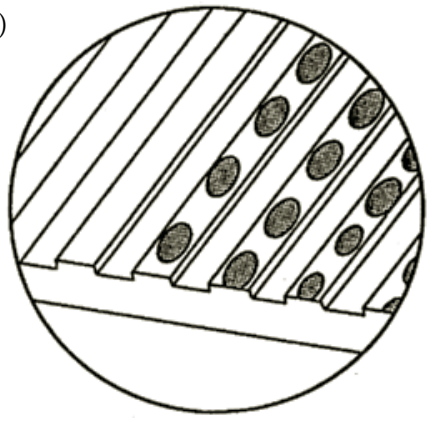

FIGURE 14.1

Pit patterns: (a) phase pit, (b) amplitude pit.

the $\mathrm{OB}$ is focused to a spot on the disk. The spot size is proportional to the wavelength $\lambda$ of the laser beam and inversely proportional to the NA of the OB.

The NA is given by the sine of the angle $\theta$ between the optical axis and the marginal rays:

$$
N A=n \sin q
$$

where $n$ represents the refractive index of the medium in object space. The fullwidth-at-half-maximum (FWHM) intensity diameter of the beam spot $\left(D_{\mathrm{s}}\right)$ on the disk is expressed as

$$
D_{\mathrm{s}}=k \frac{1}{\mathrm{Na}}
$$

where $k$ represents a constant dependent upon the light amplitude distribution at the OB pupil. If the incident beam to the $\mathrm{OB}$ is plane wave, the value of $k$ is 0.53 . When the incident beam is Gaussian or contains some aberrations, $k$ becomes larger. Since the information density of the disk is inversely proportional to the square of $D_{\mathrm{s}}$, smaller $k$ is more desirable. Supposing $k=0.53, \lambda=0.405 \mu \mathrm{m}(15.9 \mu \mathrm{in})$, and NA $=0.85$, we obtain the beam spot diameter $D_{\mathrm{s}}=0.25 \mu \mathrm{m}(9.9 \mu \mathrm{in})$. More than $1.4 \times 10^{11}$ information bits can be stored on one side of a 5.25 in optical disk using a beam of this size.

Figure 14.2 shows the playback optics for a reflective optical disk. The laser emission from the semiconductor laser (L) is reflected by a beam splitter (BS) and is incident on the 


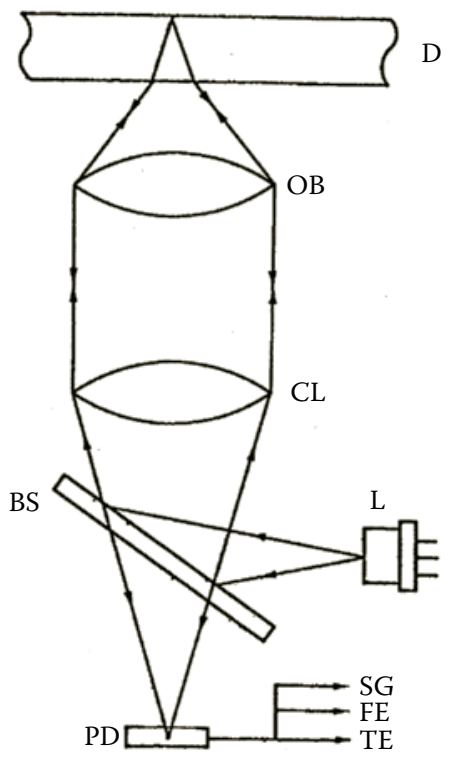

FIGURE 14.2

Playback optics.

OB through a collimating lens (CL). The wavelength $\lambda$ generally used for CD is in the range $780-800 \mathrm{~nm}$ and for DVD, 635-660 nm. The NA of the OB is generally 0.45 when used for CD and 0.6 for DVD. Next generation optical disks will have a wavelength of $405 \mathrm{~nm}$ and a NA of 0.85 . The OB aperture limits the spatial frequency response of the optical system.

The laser beam reflected from the disk is intensity modulated by the pits prior to a second pass through the OB. Part of the return beam is transmitted through the BS and is incident on the photodetector (PD). The SG, focus error signal (FE), and tracking error signal (TE) are generated from the PDs.

Reflective or transmissive mode systems can be constructed, but the reflective mode is used in most optical disk systems. In the transmissive mode, a second optical pick-up with the PDs must be positioned on the other side of the disk, complicating the design of the drive. Another problem is that the pits must be very deep and replication becomes more difficult, which leads to degradation of signals during read. A third problem with the transmission mode is difficulty in obtaining a good FE with a satisfactory $\mathrm{S} / \mathrm{N}$ ratio. Simple FE detection methods are easily achieved in reflective mode.

\subsection{APPLICATIONS OF OPTICAL DISK SYSTEMS}

\subsubsection{Read-Only Optical Disk Systems}

Four types of standardized players are available for read-only optical disks: video disk, audio disk (CD), data file disk (CD-ROM), and DVD. Among the advantages of read-only optical disks are: (1) mass replication; (2) a relatively simple optical layout as compared to write-once or rewritable system; and (3) ease of commercialization due to its use as a 
stand-alone unit. Signals in read-only optical disk systems are generally encoded with pulse width modulation (PWM), resulting in high recording density.

\subsubsection{Video Disk}

Optical video disk systems have been commercially available for many years. They have the international standard name of LV (laser vision), and use analog signal recording. Two types of disks having diameters of 30 and $20 \mathrm{~cm}$ are utilized. Rotational speed is constant at $1800 \mathrm{rpm}$ for the constant-angular-velocity (CAV) mode, and a variable rotational speed of $600-1800 \mathrm{rpm}$ is used for the constant-linear-velocity (CLV) mode. LV has relatively low recording density and is now being replaced by DVD.

\subsubsection{CD/CD-ROM}

The DAD system has been standardized using the term compact disk. The diameter of the disk is $12 \mathrm{~cm}$ (4.7 in), and the thickness of the polycarbonate protective layer is $1.2 \mathrm{~mm}$. The linear velocity can vary from 1.2 to $1.4 \mathrm{~m} / \mathrm{s}(3.9-4.6 \mathrm{ft} / \mathrm{s})$ in the CLV mode. The maximum playback time is about $75 \mathrm{~min}$, long enough to accommodate a fairly long classical music selection on a single disk. Audio signals are quantitized using 16 bits, allowing a dynamic range of $96 \mathrm{~dB}$ in playback. CD is now dominant in package media and is widely used in the music market. Using the ordinary data signal coding of the CD system, computer compatible data can be stored for use as a read-only memory of a personal computer (CD-ROM). More than 650 Mbytes of information can be stored on one side of a disk, enough to store the entire text of Encyclopedia Britannica on one side of a CD-ROM disk. Most personal computers are equipped with a CD-ROM, although DVD drives are rapidly replacing the $\mathrm{CD}$ drives in this application.

\subsubsection{DVD}

The successor to the CD is the integrated DVD optical disk system. The DVD specifications for Read-Only Disk were issued in 1996, followed by Rewritable (ver. 1.0) in 1997, Rewritable (ver. 2.0), and Re-recording (ver. 1.0) in 1999, and DVD-R for General (ver. 2.0) in 2000. These DVD systems are all integrated into DVD for Multi. The DVD disk has a storage capacity of 4.7 Gbytes, and more than 135 min of MPEG2 video signal can be stored on one side of a 12-cm disk. The DVD has overtaken almost all use of LD and CDV in video and music videos.

\subsubsection{Write-Once Disk Systems ${ }^{7-9}$}

Write-once disks have been commercially implemented in applications such as archival data memory devices for computers, document storage, and picture filing systems. Polycarbonate (PC) is being used to form injection molded disk substrates. The recording mechanism of the disk may be (1) phase-changing; (2) hole-burning; and (3) bubble-forming. In Figure 14.3, the pits formed by these different recording methods are schematically shown.

Signal pits are recorded by irradiation with a semiconductor laser focused to a spot less than 0.3 up to $1 \mu \mathrm{m}$ in diameter. This irradiation increases the temperature of the recording medium to about $200-600{ }^{\circ} \mathrm{C}\left(392-1272{ }^{\circ} \mathrm{F}\right)$, and the recording takes place as the result of the consequent physical or chemical change of the medium. 

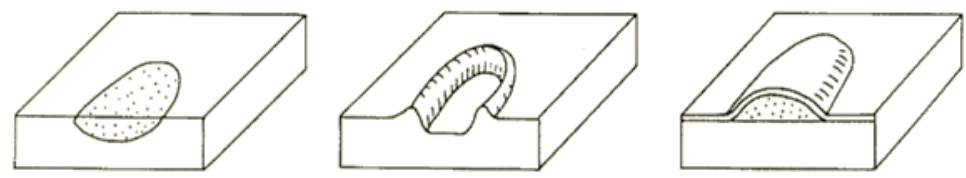

FIGURE 14.3

Pits formed by different recording methods.

\subsubsection{CD-R}

Write-once disk systems can be used for archival storage of large data files. The removability of optical disks and the standardization of products provide a broad range of application. CD-R is currently the most commonly used write-once disk. Typical specifications of CD-R disk systems are shown in Table 14.1.

\subsubsection{Erasable Optical Disk Systems}

Two major families of erasable media are available: PCR and MO. Data recording on PCR media is accomplished by inducing a transition from a crystalline phase to an amorphous phase. Differences in reflectivity of the two phases allow signal playback.

MO recording is accomplished by establishing the magnetization of a mark by heating it in the presence of a magnetic field. Read back utilizes the polarization change of the laser beam induced by magnetic modulation according to the Kerr effect. The principal characteristics of erasable disks are shown in Table 14.2. The overwrite mechanism of a PCR disk is easy to design. However, reversibility is better in MO disks. The MO disk drive requires a complicated system for applying the write and erase magnetic fields, which have opposite polarities.

\subsubsection{PCR Disk}

Figure 14.4 shows PCR optical data file drives (DVD-RAM). Figure 14.5 shows the principle of the direct overwriting mechanism for the PCR disk. The laser intensity at the disk is modulated, in correspondence with the pit pattern to be recorded, between the maximum level (A) and the intermediate level (B) as illustrated in Figure 14.5a. At exposure level (A), the material reaches a melting temperature of over $600^{\circ} \mathrm{C}$. Rapid quenching forces the material to remain in the amorphous phase, giving low surface reflectivity. Exposure level (B) heats the material to about $400{ }^{\circ} \mathrm{C}$, allowing rapid crystallization to proceed, giving an increased reflectivity. Figure $14.5 \mathrm{~b}$ is a schematic illustration of the overwriting operation.

\subsubsection{MO Disk ${ }^{10,11}$}

In MO disks, a light beam is directed at a magnetic material to record or erase information. The underlying principle is the utilization of a temperature-dependent change of magnetic properties. There are several methods, including Curie point recording and compensation point recording, which can be used for recording. Figure 14.6 is an elementary illustration showing the principle of Curie point recording. In this example the initial magnetization of the recording layer is uniformly oriented in a given direction, as shown in Figure 14.6a. When a limited area of the recording layer is irradiated with light sufficient in intensity to heat it to a temperature above the Curie point $T_{c}$, the magnetization of the local area 
TABLE 14.1

Specifications of CD-R Disk Systems

\begin{tabular}{lll}
\hline Items & Unit & \multicolumn{1}{c}{ Specifications } \\
\hline User data capacity & Mbytes & $\sim 650$ \\
Disk diameter & $\mathrm{Mm}$ & 120 \\
NA of OB & & 0.5 \\
Wavelength & $\mathrm{Nm}$ & $775-795$ \\
Wavefront distortion of pick-up & $\Lambda$ & $<0.050$ \\
Recording power & $\mathrm{mW}$ & $4<\mathrm{Po}<8$ \\
Playback power & $\mathrm{mW}$ & $<0.7$ \\
Thickness of disk substrate & $\mathrm{Mm}$ & 1.2 \\
Rim intensities & & Tangential $0.14 \pm 0.04$ \\
& & Radial $0.7 \pm 0.10$ \\
\hline
\end{tabular}

TABLE $\mathbf{1 4 . 2}$

Characteristics of Erasable Optical Disks

\begin{tabular}{lll}
\hline & \multicolumn{1}{c}{ PCR } & \multicolumn{1}{c}{ MO } \\
\hline Recording and erasing mode & Phase change & Change of magnetization \\
Read & Change of amplitude & Change of polarization \\
Material of medium & Te-Ge-Sb & Tb-Fe-Ni-Co \\
Overwrite mechanism & Simple & Complicated \\
Magnetic field & Not required & Required \\
Reversibility & Fair & Good \\
Required power & High & Medium \\
\hline
\end{tabular}

PCR, phase-change rewritable; MO, magneto-optical.

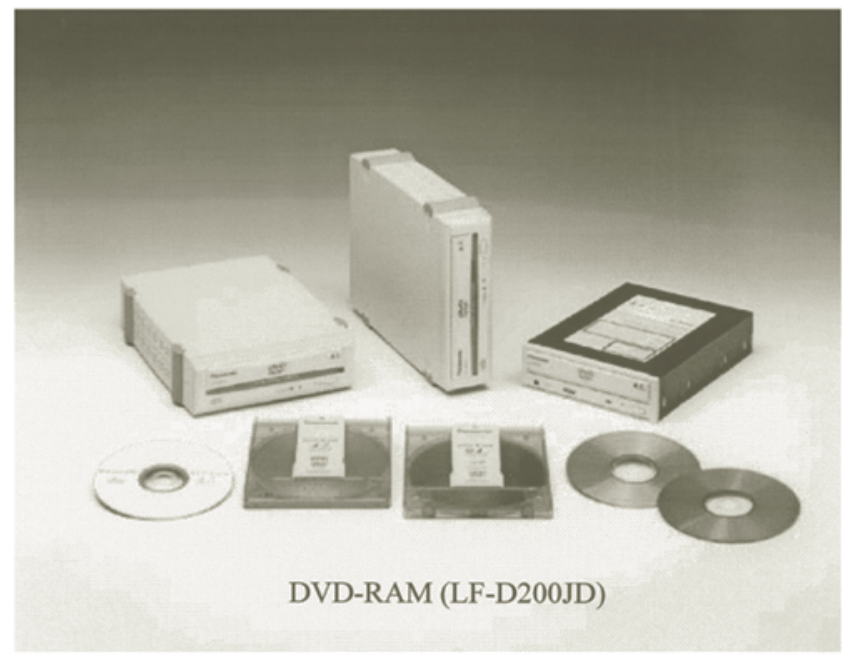

FIGURE 14.4

Optical data file drives (DVD-RAM). 
(a)

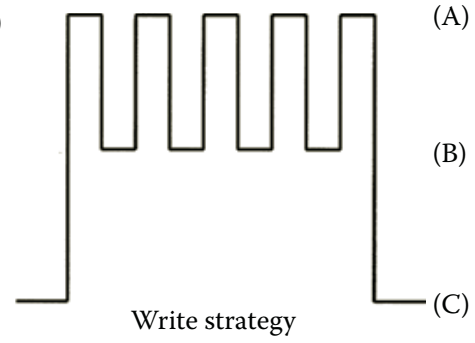

(b)

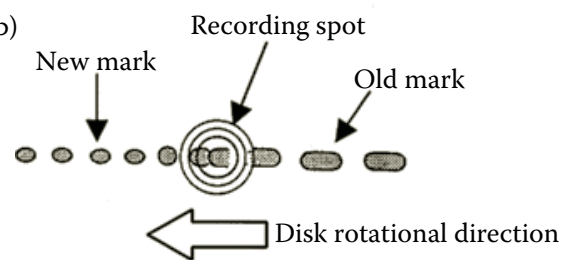

Overwriting marks

(A)

(B)

FIGURE 14.5

Principle of the direct overwriting mechanism for the PCR disk: (a) Write strategy, (b) Overwriting marks.

is lost, as shown in Figure 14.6b. When the exposure is discontinued, the temperature of the recording layer falls below $T_{c}$. The exposed area is remagnetized, but the direction of this magnetization coincides with the direction of the applied external magnetic field. Therefore, if the external magnetic field is applied in a direction opposite that of the original magnetization of the recording layer, as shown in Figure 14.6b, a magnetic domain different from the surrounding area remains, as shown in Figure 14.6c, enabling the recording of binary information. For reading the signal, the recording layer is irradiated with a laser light of low power. The polarization rotations of the reflected beam from the signal surface and the land surface are in opposite directions, as shown in Figure 14.6c. These beams are detected with a polarization analyzer to obtain the read signal. To erase the information, a selected area is again heated to a temperature above the Curie point, as shown in Figure 14.6d. The direction of the external magnetic field is reversed from that for recording.

In signal readout, the linear polarization angle of the incident beam is set at $\theta$. The Kerr rotation angle is given by $\pm \Phi_{k}$. Referring to Figure 14.7, the differential output $I$ of the analyzer between the $x$ and $y$ directions is given by

$$
\begin{aligned}
\Delta I & =I_{0} R\left[\cos ^{2}\left(q-\Phi_{k}\right)-\cos ^{2}\left(q+\Phi_{k}\right)\right] \\
& =(1 / 2) I_{0} R \sin (2 q) \sin \left(2 \Phi_{k}\right)
\end{aligned}
$$

where $R$ is the disk reflectivity.

Since the linear polarization angle of the incident beam is $p / 4$ and $\Phi_{k} \ll 1$

$$
\Delta I \sim I_{0} R \Phi_{k}
$$

Thus, the playback signal level is proportional to the incident light intensity $I_{0}$, the disk reflectivity $R$, and the Kerr rotation angle $\Phi_{k}$. 


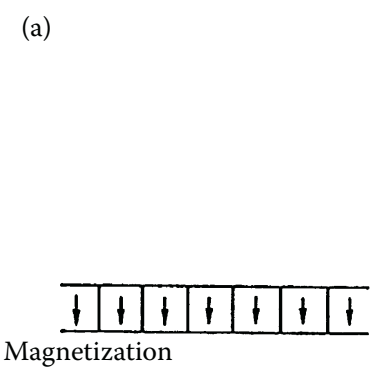

Before writing

(c)

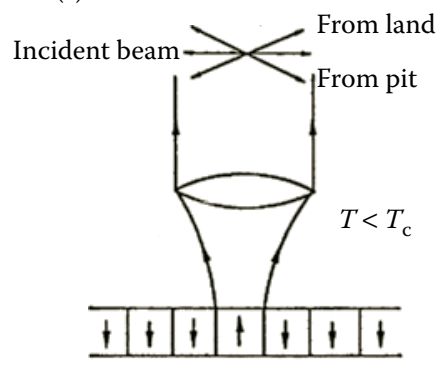

Reading

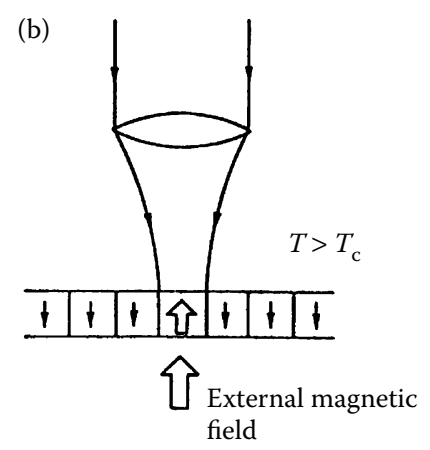

Writing

(d)

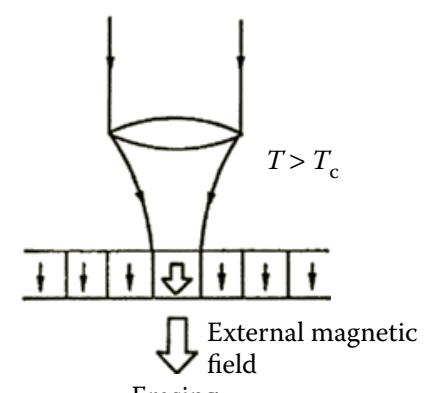

Erasing

FIGURE 14.6

Magneto-optical disk method: (a) before writing, (b) writing, (c) reading, (d) erasing.

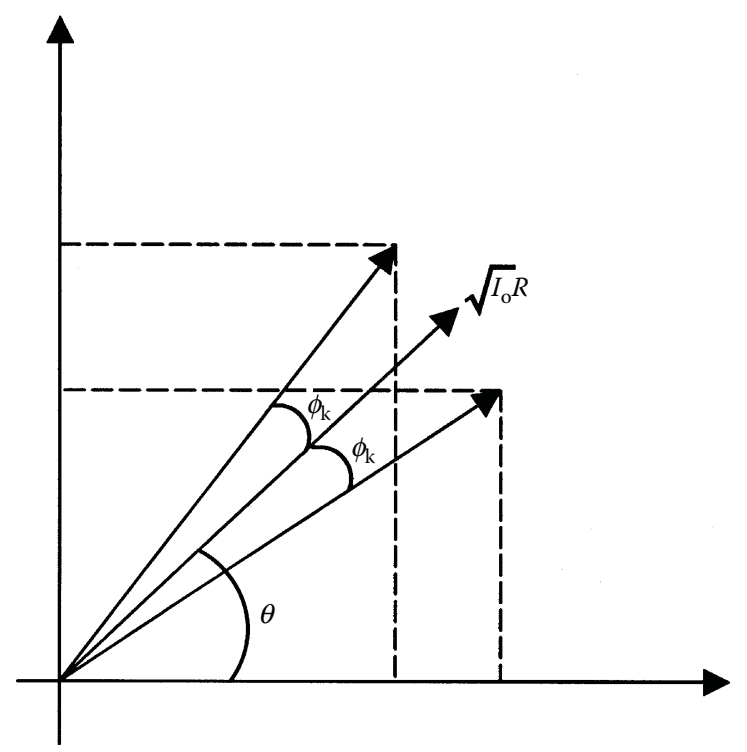

FIGURE 14.7

Readout of MO signal. 


\subsection{BASIC DESIGN OF OPTICAL DISK SYSTEMS}

\subsubsection{Pick-Up Optics}

The many types of optical disks described in the preceding sections each have optimized optical pick-ups. The methods of design for the optics and mechanics of a writable optical pick-up will be described in this section.

The following factors determine the quality of read/write signals.

1. Frequency characteristics of signals

2. Cross talk from the adjacent tracks, which degrades read/write signals

3. Carrier-to-noise ratio (CNR) of read/write signals

4. Errors rate in read/write signals

Factors 1 and 2 are mainly dependent on the wave aberrations of the optics. Factor 3 is as much associated with the characteristics of elements such as the semiconductor laser, detector and electronics as with wave aberrations, and factor 4 is mainly dependent on defects in the disk.

\subsubsection{Optical Layout}

The schematic construction of the optics for a writable optical pick-up is shown in Figure 14.8. In this example, the astigmatic method is used for detecting the focusing signal and

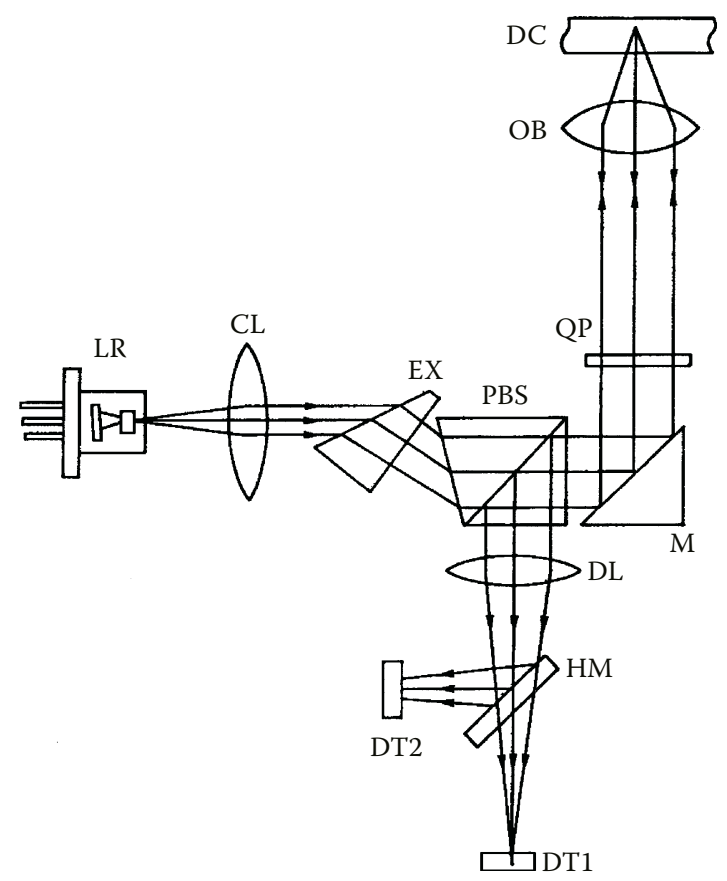

FIGURE 14.8

Schematic construction of a writable optical pick-up. 
the push-pull (PP) method is used for detecting the tracking signal. The laser beam emitted from the semiconductor laser (LR) has a near-field pattern elongated in the direction of the active layer of the laser and is polarized in the same direction. The beam waist in this direction lies within the laser, and the beam waist in a direction perpendicular to the above direction is situated at the end facet of the laser active layer. The beam emergent from the laser is therefore anamorphic, and its far-field distribution is elliptical in cross section with an ellipticity of 2 to 3 . To correct this elliptical distribution, it is necessary to use a one-dimensional afocal system after the CL, consisting of two cylindrical lenses or a wedge prism. With a single wedge prism, the designed incident angle of the laser beam must be approximately $69-72^{\circ}$. Since the wedge prism has chromatic dispersion, a change of the wavelength of the laser results in an angular deviation of the beam. Taking this angular deviation as $a$ and the focal length of the OB as $f_{\mathrm{o}^{\prime}}$ the beam spot moves approximately by $f_{\mathrm{o}} \cdot \Delta q$ on the disk (DC). Using a single BK7 wedge prism with the incident angle $72^{\circ}$, an $\mathrm{OB}$ with focal length $4.5 \mathrm{~mm}$ and wavelength $0.78 \mu \mathrm{m}$, the beam spot displacement on the disk is approximately $0.073 \mu \mathrm{m}$ for a change of $1 \mathrm{~nm}$ in wavelength. Therefore, the optics should be designed such that the direction of this movement will not cause a track offset. For this reason it is good practice to use two wedge prisms as illustrated in Figure 14.8. In the case of the playback-only optical pick-up, the influence of the elliptical and astigmatic beam can be small at the cost of beam utilization efficiency.

In Figure 14.8, the laser beam transmitted through a polarizing beam splitter (PBS) as a p-polarized beam passes through the $\lambda / 4$ plate $(\mathrm{QP})$ to become a circularly polarized beam, which is incident on the OB. The beam emerging from the OB is incident on the disk (DC) to form a beam spot for recording and reproducing the signals. The beam reflected at the disk enters the $\mathrm{OB}$ and again passes through the $\lambda / 4$ plate $(\mathrm{QP})$ to become an s-polarized beam and is reflected to the detection lens (DL) by the PBS. The beam emergent from the DL is partially reflected by a half-mirror (HM) and incident on the detector (DT2) for PP tracking signal detection. Because the convergent beam passing the HM is astigmatic, it is received by a quadrant detector (DTI) to give a focusing signal. The data signal is retrieved by sum of the output from both detectors (DT1 and DT2). The astigmatic focusing and PP tracking methods will be described in detail later.

\subsubsection{Influence of Intensity Distribution}

The intensity distribution of the beam incident on the OB is dependent on the beam divergence angle distribution of the semiconductor laser. With the OB aperture radius being standardized as unity and the intensity distribution of the incident beam assumed to be $\exp \left(-a r^{2}\right)$, the amplitude distribution is given by the Fourier-Bessel transform:

$$
g(s)=\int \exp \left(-a r^{2}\right) J_{0}(s r) r \mathrm{~d} r
$$

with $s=2 p n R / \lambda f_{0}$, where $f_{0}$ is the focal length of the OB and $R$ is the polar coordinate in the focal plane. Integration gives (Appendix A, Equation 14.A4):

$$
g(s)=\sum_{n=0}^{\infty} 2^{n} a^{n} e^{-a}\left(\frac{2 J_{n+1}(s)}{s^{n+1}}\right)
$$

Since $\otimes=0$ for plane-wave incidence,

$$
\left.g(s)\right|_{a=0}=\frac{2 J_{0}(s)}{s}
$$


This is the well-known Airy distribution. When $a=1$, the beam intensity distribution around the OB aperture is $1 / e^{2}$. Figure 14.9 shows the intensity distribution of the $|g(s)|^{2}$ beam spot with various values of $a$. It is apparent from Figure 14.9 that when $a=1$, the FWHM of the beam spot is increased by about $10 \%$ (relative to $a=0$ ), and the peak of the side-lobe diffraction ring is made sufficiently small.

In order for the reproduced signal to have satisfactory frequency characteristics, the value of $\alpha$ in the signal direction must be in the range of $a \ll 1$. On the other hand, in the direction perpendicular to the signal direction, the cross talk from the adjacent track must be minimized. This cross talk can be small by using $\alpha$ close to 1 . Therefore, the spot on the disk need not be truly round, but an improved frequency characteristic is sometimes obtained when the beam spot is elliptical with an ellipticity of about $10 \%$.

\subsubsection{Wave Aberrations ${ }^{12}$}

When root-mean-square wave aberration $W$ exists, the on-axis energy density Strehl definition (SD) of the beam spot is expressed by

$$
S D=1-k^{2} W^{2} \quad \text { with } k=\frac{2 p}{1}
$$

where $\lambda$ is the wavelength.

Figure 14.10 shows the relation between rms wave aberration and on-axis energy density. The on-axis energy density SD is a factor directly associated with reproduced signal SNR or record/reproduced signal SNR. The allowable rms wave aberration for the whole optical disk system is subject to Maréchal's criterion that the rms wave aberration is $0.070 \lambda$ when SD has decreased about $20 \%$ from the level at no aberration. The validity of the criterion has been endorsed by read/write experiments. This allowable wave aberration for the whole system must be allocated to disk thickness error and tilt error, initial optical aberration, and defocus value.

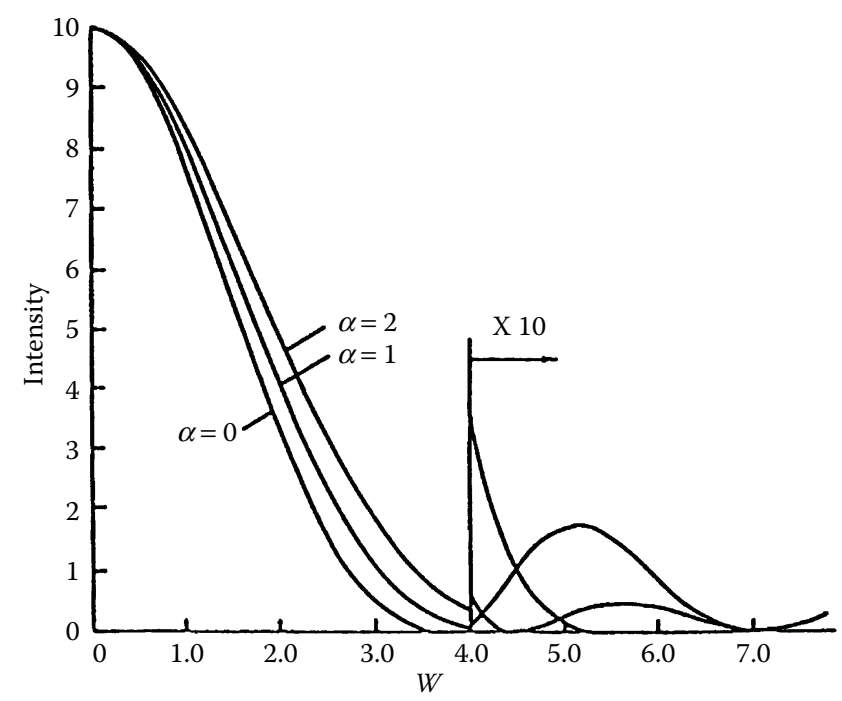

FIGURE 14.9

Intensity distributions of laser beam spot. 


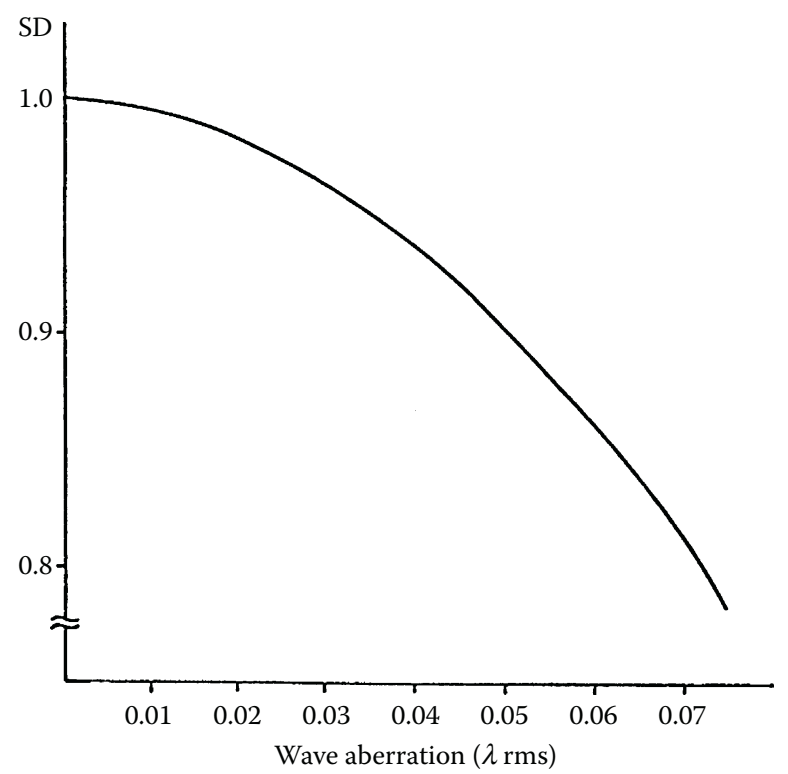

FIGURE 14.10

Wave aberrations vs. energy density on axis.

\subsubsection{Aberration Derived from Disk Substrate}

The aberration originating from the disk substrate is composed of the aberration $W_{\mathrm{ST}}$ due to the error $t$ of substrate thickness $t$ and the aberration $W_{\mathrm{TL}}$ due to the inclination $\theta$ of the substrate. These aberrations, when small, are expressed by the following equations (also Equations 14.A11 and 14.A14):

$$
\begin{aligned}
& W_{\mathrm{ST}}=\frac{\Delta t\left(n^{2}-1\right)(\mathrm{NA})^{4}}{8 \sqrt{180} n^{3}} \\
& W_{\mathrm{TL}}=\frac{t\left(n^{2}-1\right) q(\mathrm{NA})^{3}}{2 \sqrt{72} n^{3}}
\end{aligned}
$$

where NA is the NA of the $\mathrm{OB}$ and $n$ is the refractive index of the disk substrate. Figure 14.11 shows the relation between disk thickness error $t$ and wave aberration $W_{\mathrm{ST}}$ with NA and wavelength $\lambda$ as the parameters. Figure 14.12 shows the relation between disk tilt angle and wave aberration $W_{\mathrm{TL}}$ with NA and wavelength $\lambda$ as the parameter. In the usual recordable $C D$, the practical values are $\mathrm{NA}=0.5, t=1.2 \mathrm{~mm}, n=1.51, \lambda=780 \mathrm{~nm}, \Delta t=40$ $\mu \mathrm{m}$, and $\theta=4 \mathrm{mrad}$. Substituting these values, we obtain $W_{\mathrm{ST}}=0.011 \lambda$ and $W_{\mathrm{TL}}=0.017 \lambda$. In the DVD optical disk, the values are NA $=0.6, t=0.6 \mathrm{~mm}, n=1.51, \lambda=650 \mathrm{~nm}, \Delta t=16 \mu \mathrm{m}$, and $\theta=3.9 \mathrm{mrad}$ for the same values of $W_{\mathrm{ST}}=0.011 \lambda$ and $W_{\mathrm{TL}}=0.0172 \lambda$. The plot for higher $\mathrm{NA}=0.85$ and short wavelength $\lambda=405 \mathrm{~nm}$ is shown as a reference. Thus the tolerance of the tilt angle for the DVD disk is almost the same as for the CD, as contrasted with the tolerance of the thickness being small. 


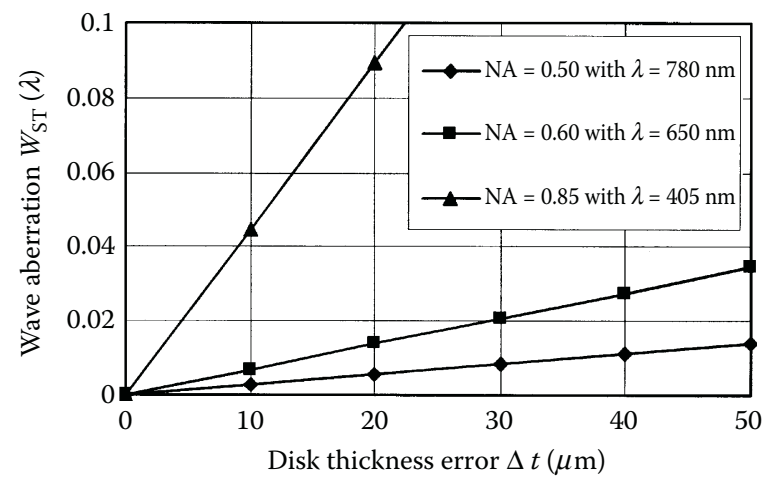

FIGURE 14.11

Wave aberrations versus disk thickness error.

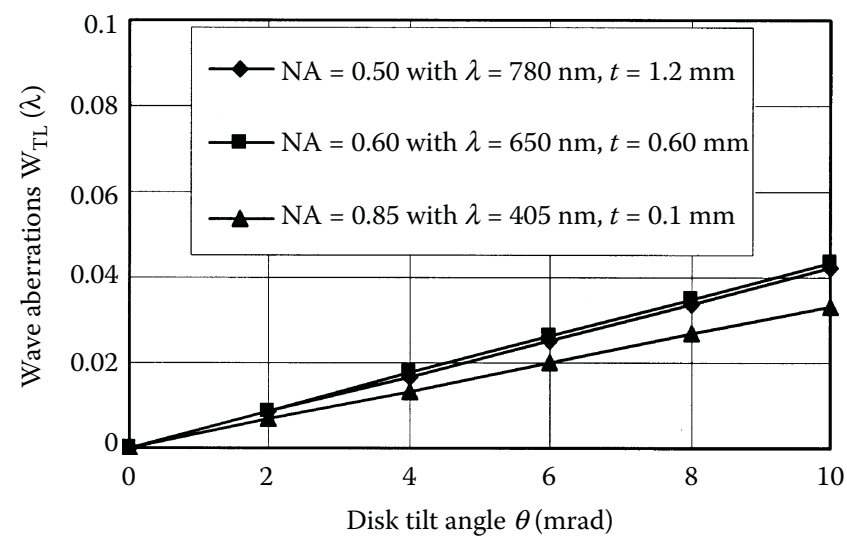

FIGURE 14.12

Wave aberrations versus disk tilt angle.

\subsubsection{Wave Aberrations of Optical Components}

Because mass-produced items are used for the disk optical components, the influence of variations in wave aberrations cannot be disregarded. Of all the components of the optical pick-up, the OB and the CL have the largest wave aberrations. Both the OB and the CL usually are aspherical pressed glass (APG) available on a mass production basis. Figure 14.13 shows an example of a mass-produced aspherical OB. Figure 14.14 shows the wave aberrations of a typical APG OB as measured with a Fizeau interferometer. The wave aberrations of prism systems are generally small, but as the number of prisms increases, the allowance for the entire pick-up is consumed.

\subsubsection{Aberration Due to the Semiconductor Laser}

Semiconductor lasers are generally astigmatic, and as this astigmatism is propagated and focused on the disk, variations in frequency characteristics may occur according to relative directions on the disk, or there may be reduced focusing latitude. In the record-playback optics, the electromagnetic emission from a semiconductor laser is passed through a CL 


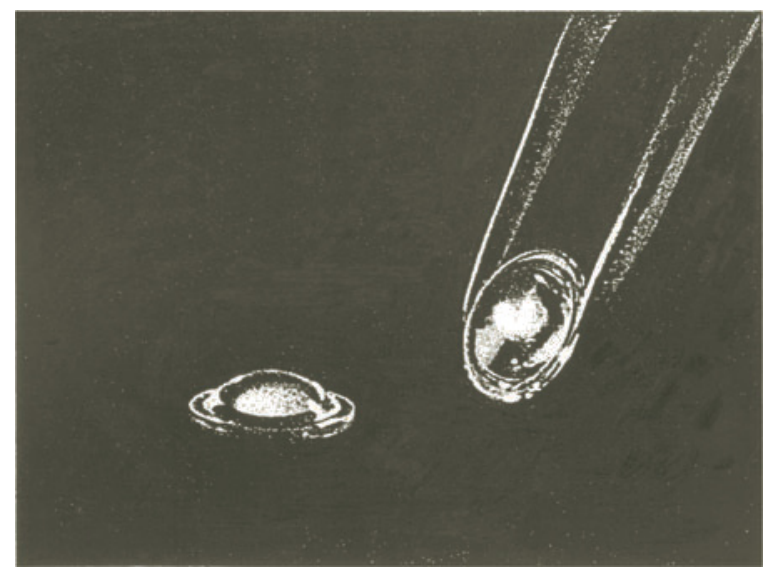

FIGURE 14.13

Mass-produced aspherical objective lens.

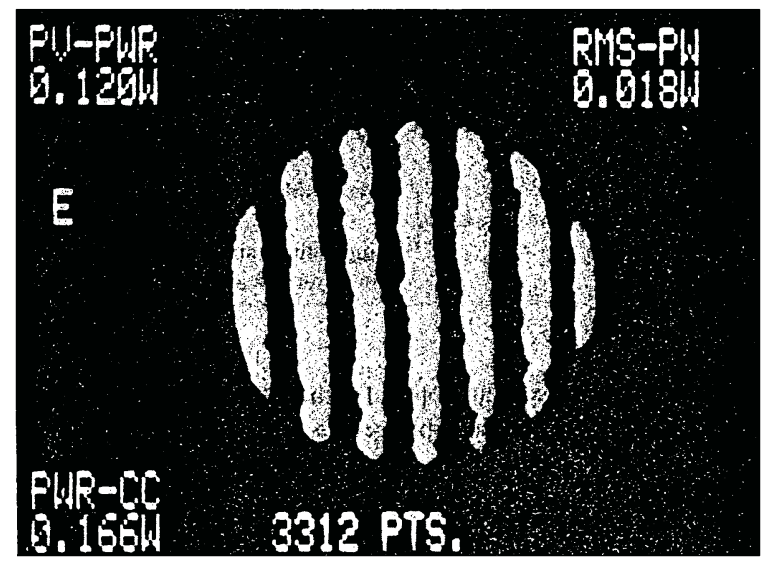

\section{FIGURE 14.14}

Wave aberrations of a typical APG objective lens.

to yield a beam of substantially parallel rays, which is then converted by an anamorphic beam expander to a beam having substantially isotropic distribution.

The correction for astigmatism is carried out concurrently in this stage. If stationary prism is used for correction, the astigmatism generated at an angle of $45^{\circ}$ with the prism cannot be corrected. Therefore, when the semiconductor laser is mounted at an angle $\theta$ with the horizontal direction of the anamorphic expander prism, there occurs a residual astigmatism. The residual wave aberration $W_{\mathrm{LA}}$ due to this astigmatism is expressed as (Equation 14.A20)

$$
W_{\mathrm{LA}}=\frac{\tan q \Delta_{\mathrm{L}}\left(\mathrm{NA} \mathrm{A}_{\mathrm{C}}\right)^{2}}{\sqrt{6} \cos ^{2} q}
$$

where $\mathrm{NA}_{C}$ is the NA of the CL and ${ }_{L}$ is the astigmatism of the semiconductor laser. Assuming that the allowable wave aberration dependent on the semiconductor laser is $0.010 \lambda$, the NA of the CL is less than 0.25 and the astigmatism of the semiconductor laser 
is less than $8 \mu \mathrm{m}(0.32 \mathrm{mil})$, and the allowable angle $\theta$ of the semiconductor laser is found from Equation 14.11 to be

$$
q \leqq \pm 4^{\circ}
$$

\subsubsection{Defocus}

The factors responsible for defocus in an optical system may be classified as in Table 14.3. The relationship between the amount of defocus $\varepsilon$ and the maximum optical path difference ${ }_{D F}$ of the wavefront can be found from Figure 14.15:

$$
\Delta_{\mathrm{DF}}=\frac{e(N A)^{2}}{2}
$$

Using the relationship between maximum optical path difference ${ }_{D F}$ and wave aberration $W_{\mathrm{DF}^{\prime}}$ wave aberration can be expressed as

$$
W_{\mathrm{DF}}=\frac{\Theta(N A)^{2}}{4 \sqrt{3}}
$$

TABLE 14.3

Defocusing Factors

\begin{tabular}{lll}
\hline Defocus & Static defocus & Initial setting error \\
\hline & Aging error \\
& Dynamic defocus & Servo residual error \\
& Temperature- and humidity-related error \\
\hline
\end{tabular}

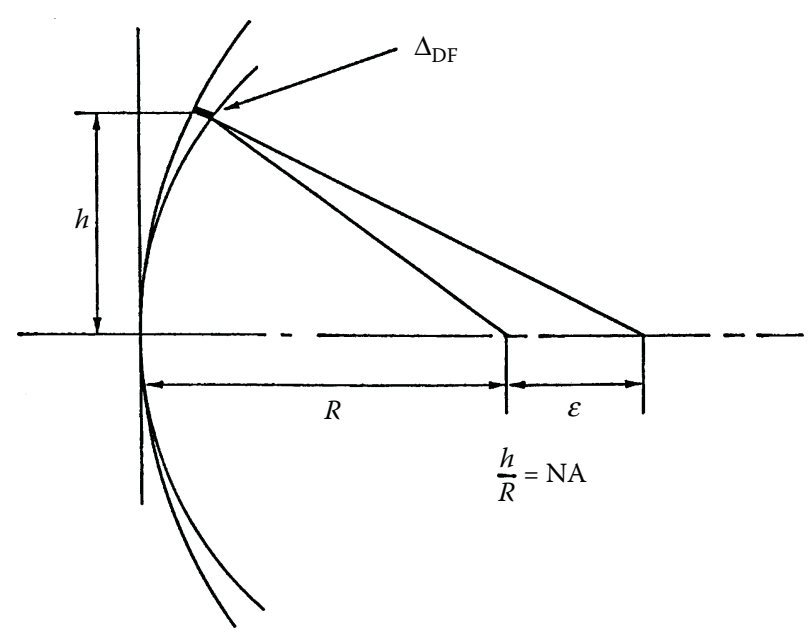

FIGURE 14.15

Optical path difference versus defocus. 
For initial focus setting, the use of a diffraction grating having a spatial frequency near one-half of the cutoff frequency $2 \mathrm{NA} / \mathrm{I}$ of the disk optics is advantageous, for the influence of defocus is then most pronounced. Because the track pitch of the optical disk is usually the space frequency in the vicinity, the position of best focus is where the modulation by the track is maximal. By this adjustment, the setting error can be reduced to less than $\pm 0.14 \mu \mathrm{m}$. Thus, with an optical disk of NA $=0.6$ and $\lambda=650 \mathrm{~nm}$ and a defocus of $+0.14 \mu \mathrm{m}$, the wave aberration is $W_{\mathrm{DF}}=0.0111$.

\subsubsection{Allowable Wave Aberration}

Table 14.4 shows the typical wave aberration classified by causative factors. These wave aberrations can be integrated into the system allowance limit of $0.070 \lambda \mathrm{rms}$ as a totality. Since most of the factors responsible for wave aberrations are independent by nature, it is possible, in the actual design of an optical pick-up, that the allowable aberration value of each optical component is fairly liberal, as shown in Table 14.4.

\subsubsection{Optical Pick-Up Mechanism}

\subsubsection{Optical Pick-Up Construction ${ }^{13,14}$}

The optical pick-up generally consists of an optical base forming the optics assembly and an actuator for allowing the OB to follow the disk plane and tracking groove.

A typical optical pick-up construction for DVD is shown in Figure 14.16. Laser and PDs are combined on one silicon substrate. The beam emitting from the laser is initially parallel to the silicon surface and is then reflected by an engraved mirror to become perpendicular to the silicon surface. A polarizing hologram, shown in Figure 14.17, is used as a BS. It is transparent for the p-polarized beam emitted from the laser and diffractive for the s-polarized beam reflected from the disk. The p-polarized beam becomes an s-polarized beam as a consequence of the double pass through the quarter-wave plate. PDs formed on the silicon surface are located on both sides of the laser, and receive the beam diffracted by the polarizing hologram. The environmental resistance and reliability of the system are greatly enhanced when the number of reflective surfaces is minimized throughout

TABLE 14.4

Factors Responsible for Wave Aberrations and Amounts of Aberrations for DVD

\begin{tabular}{|c|c|c|}
\hline \multicolumn{3}{|c|}{ System allowance limit $0.070 \lambda$} \\
\hline \multirow[t]{4}{*}{$\overline{\text { Disk } 0.028 \lambda}$} & Thickness error $< \pm 1.6 \mu \mathrm{m}$ & $\leq 0.011 \lambda$ \\
\hline & Tilt $< \pm 4$ mrad & $\leq 0.017 \lambda$ \\
\hline & Semiconductor laser & $\leq 0.010 \lambda$ \\
\hline & Objective lens & $\leq 0.035 \lambda$ \\
\hline \multirow[t]{5}{*}{ Head $0.054 \lambda$} & Collimating lens & $\leq 0.025 \lambda$ \\
\hline & Wedge prism & $\leq 0.014 \lambda$ \\
\hline & PBS & $\leq 0.020 \lambda$ \\
\hline & $\lambda / 4$ plate & $\leq 0.020 \lambda$ \\
\hline & Perpetual change & $\leq 0.011 \lambda$ \\
\hline \multirow[t]{3}{*}{ Defocus $0.036 \lambda$} & Initial setting error & $\leq 0.011 \lambda$ \\
\hline & Servo residual error & $\leq 0.023 \lambda$ \\
\hline & Temperature-dependent error & $\leq 0.023 \lambda$ \\
\hline
\end{tabular}




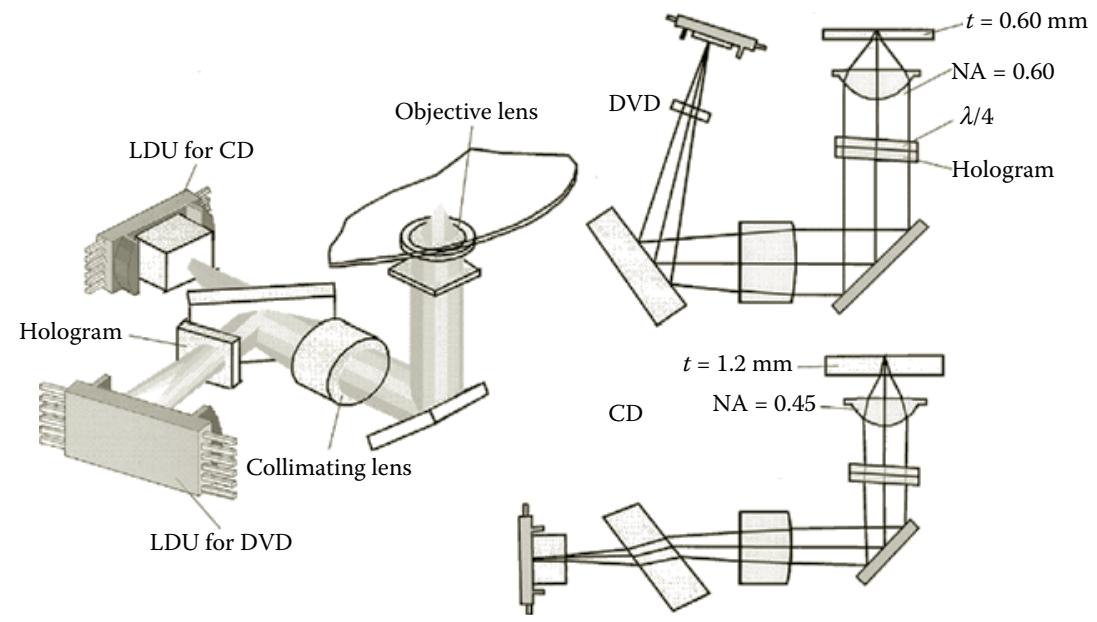

FIGURE 14.16

Optical pick-up for DVD.

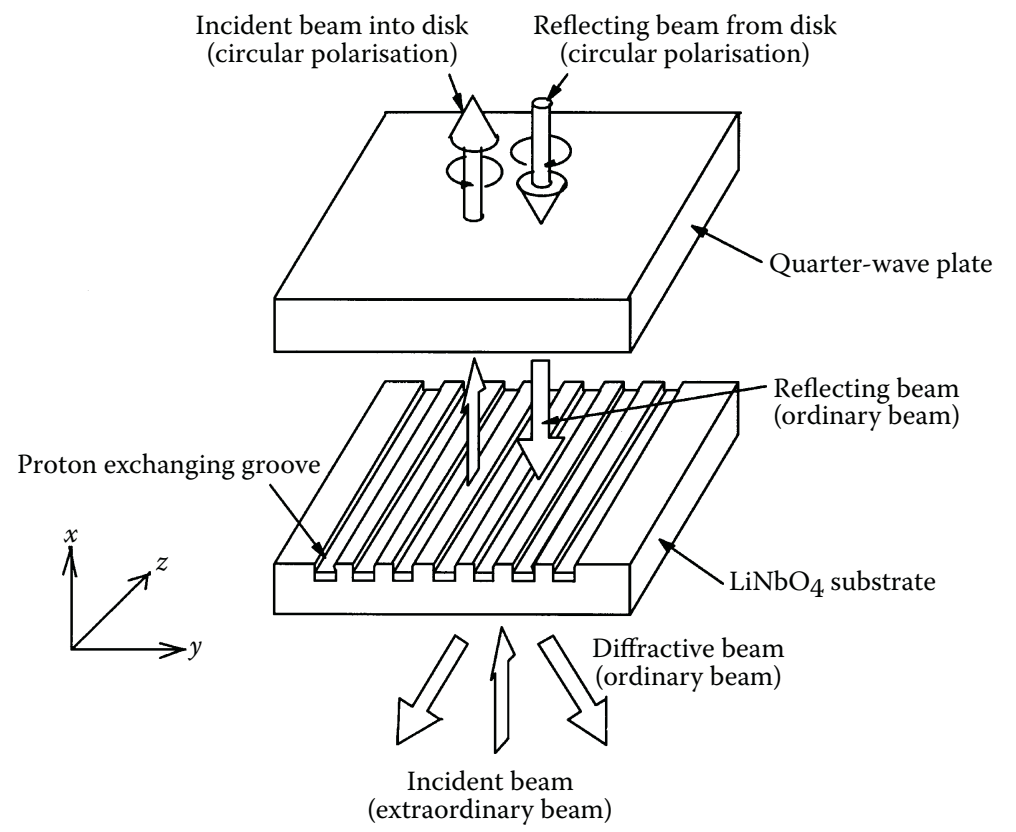

FIGURE 14.17

Polarizing hologram.

the optical path from the laser to the OB. Thus the construction with the integrated laser detector unit (LDU) is advantageous for reliability. Figure 14.18 is a view showing a LDU for a DVD player.

The optical base has a three-point support structure that enables a two-dimensional adjustment of the tilt angle of the optical pick-up. The axis of the OB is aligned perpendicular to the disk plane by mechanical adjustment, and the tilt of the optical base or the angle of inclination of the actuator must be adequately adjusted. The actuator and the optical 


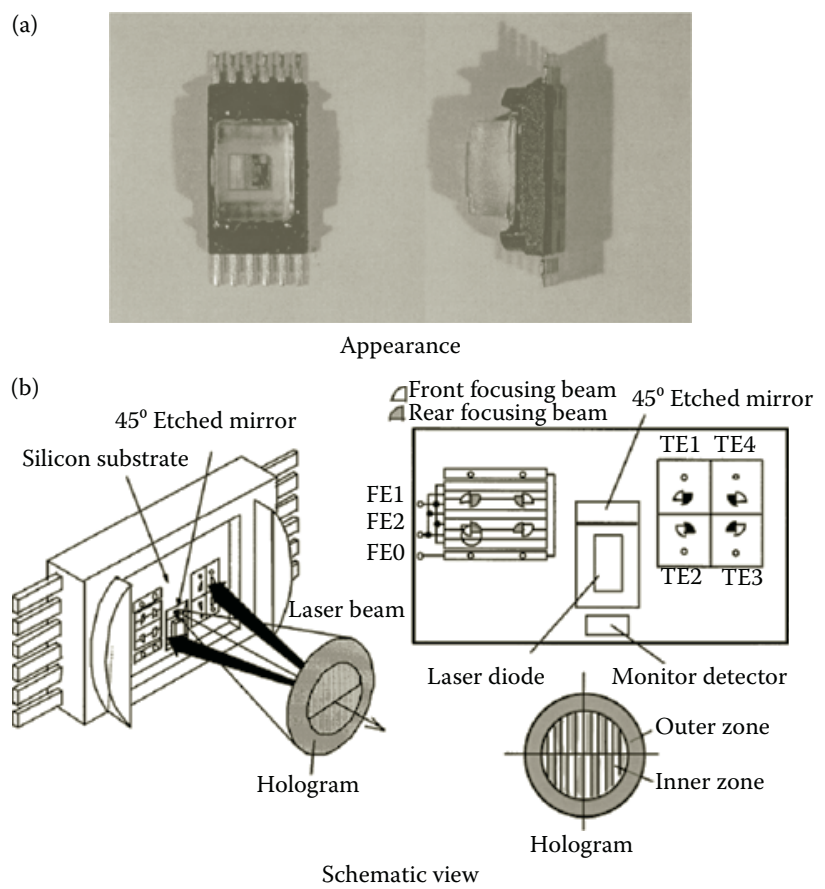

\section{FIGURE 14.18}

Integrated laser detector unit for DVD: (a) Appearance, (b) Schematic view.

base are provided with a convex and a concave spherical surface, respectively, whereby tilt correction can be made in two dimensions by means of couple of screws and springs. When the center of the sphere is aligned with the focal point of the OB, there is no transverse shift of the beam as it passes through the OB.

\subsubsection{Actuator}

The actuator has both a focusing drive mechanism for following the axial position of the disk and a tracking drive mechanism for following the track on the disk. The actuator must include a balanced combination of these two mechanisms, and must be designed in such a manner that there will be a minimum of interference between the mechanisms. The essential conditions that must be satisfied in the design of an actuator are:

1. Satisfactory frequency characteristics

2. High acceleration characteristics

3. High current sensitivity

4. Broad dynamic ranges for both focusing and tracking

Two actuator constructions satisfying these criteria are shown in Figure 14.19. The wiresuspended actuator ${ }^{15}$ in Figure 14.19a is quite simple in construction and can be moved in the focusing and tracking directions by driving the center of gravity of its movable segment. Moreover, reliability is high because the four wires can be utilized as leads to the coils. The rotational actuator in Figure $14.19 \mathrm{~b}$ is characterized by a small tilting angle of the critical axis and a large focusing dynamic range. 
(a)

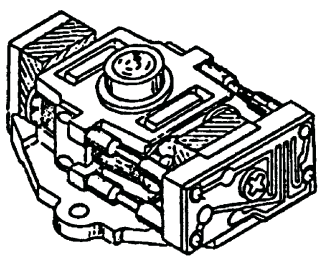

(b)

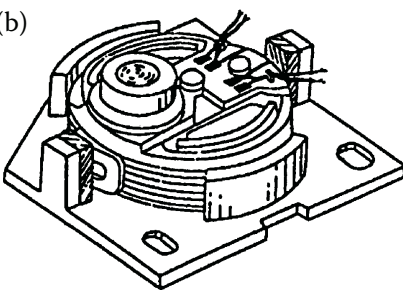

FIGURE 14.19

Two different types of actuator: (a) wire-suspended and (b) rotational.

The first-order resonant frequency $f_{0}$ of the wire-suspended actuator is

$$
f_{0}=2 p \sqrt{\frac{K}{m}}
$$

where $K$ is a spring constant and $m$ is a movable mass. As a rule of thumb, the dynamic frequency range of an actuator is approximately from the level of the basic disk rotation frequency to the peak level of high-order resonant frequency. The larger this dynamic range value is, the larger is the servo gain that can be obtained.

\subsection{SEMICONDUCTOR LASER}

\subsubsection{Laser Structure}

\subsubsection{Operating Principles of an Al-Ga-As Double Heterojunction Laser ${ }^{16}$}

The energy band diagram of a double heterojunction semiconductor laser is shown in Figure 14.20. This laser consists of three layers having dissimilar energy gaps Eg, with increased energy gaps for the n-type and p-type cladding layers, which are on both sides of the active layer. As a photon $h v g_{2}$ corresponding to the active layer energy gap $\operatorname{Eg}_{2}$ $\left(\mathrm{Eg}_{2}=h v g_{2}\right)$ passes through the active layer, the electrons in the conduction band drop into the positive holes in the valence band to trigger a stimulated emission in phase with the incident photon. As a current $I_{p}$ in the normal direction is passed through this diode, the probability of the presence of electrons in the active layer 2 is increased by the energy barrier $E_{c^{\prime}}$ the conduction band. On the other hand, in the valence band, the probability of the presence of positive holes in the active layer 2 is increased by the energy barrier $E_{\mathrm{v}}$ to cause a population inversion in the active layer 2. In the active layer, therefore, the conduction band becomes full of electrons normally absent at thermal equilibrium, and the probability of recombination of electron-hole pairs with stimulated emission is increased. An incoming photon into the active layer is thereby amplified. The feedback mirrors at both ends of the active layer constitute a resonant cavity, and as the amplification surpasses the losses within the resonance cavity laser emission takes place.

\subsubsection{High-Power Laser Technology ${ }^{17}$}

Low-current and high-temperature operating laser diode of $650 \mathrm{~nm}$ AlGaLnP with a real refractive index guided self-aligned (RISA) structure is schematically illustrated in 
Figure 14.21. The RISA structure is characterized by an AlInP current blocking layer, which leads to small internal loss in the waveguide and substantially reduces operating carrier density. The resultant operating current for $950 \mathrm{~mW}$ continuous wave at $70^{\circ} \mathrm{C}$ is less than $100 \mathrm{~mA}$. The RISA laser is produced by two steps of MOCVD growth. In the first MOCVD growth, an n-GaAs buffer layer, a cladding layer, the MQW active region, the optical confinement layer, the current blocking layer, and a nondoped $\mathrm{GaAs}(0.01 \mu \mathrm{m})$ are successively grown. Then, the stripe region for the current path is formed by chemical etching. In the second MOCVD growth, the cladding layer, buffer layer, and contact layer are grown. The cavity length is $500 \mu \mathrm{m}$. Front and rear facets are coated to obtain reflectivities of $4 \%$ and $90 \%$, respectively.

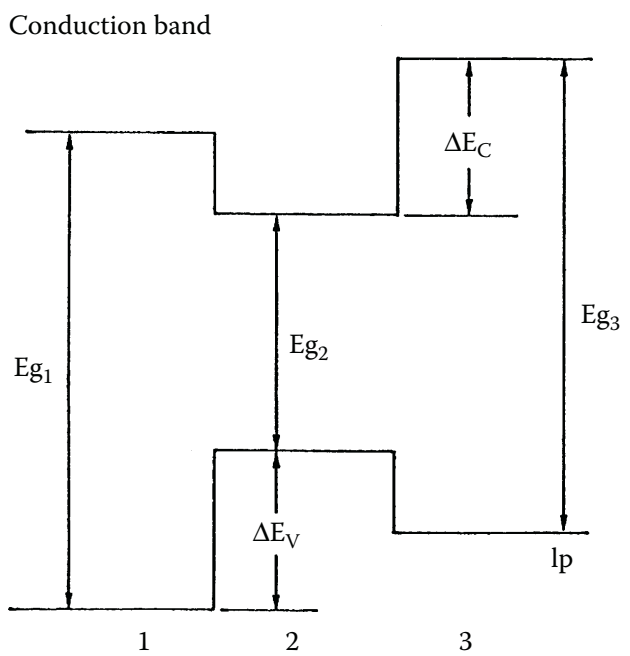

Valence band

FIGURE 14.20

Energy band diagram.
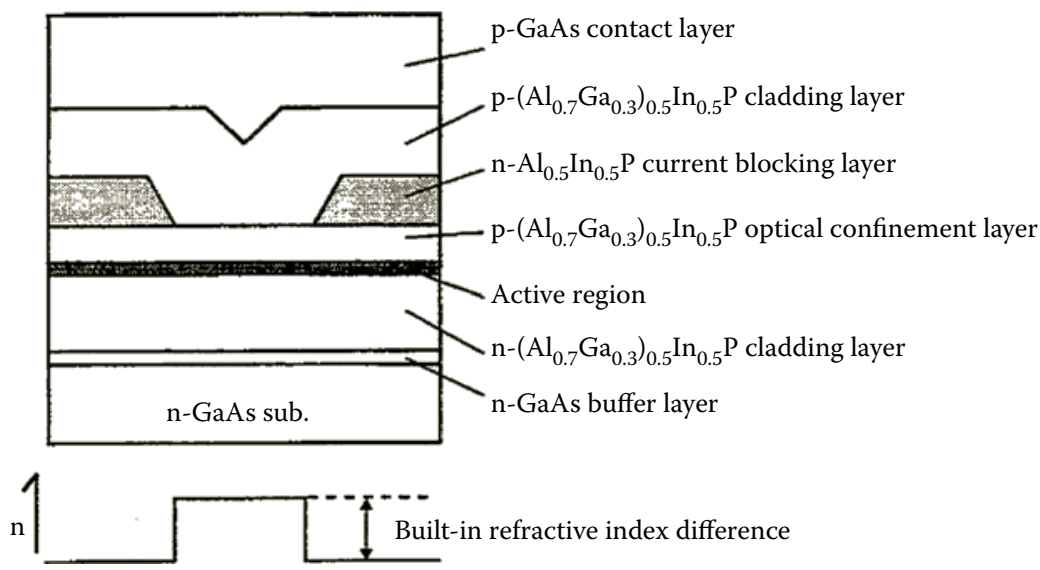

FIGURE 14.21

Schematic drawing of the RISA laser structure. 


\subsubsection{Astigmatism of the Laser}

There are two categories of semiconductor lasers: gain-guided and index-guided. In a gainguided laser the direction of beam propagation is not perpendicular to the wavefront. This mismatch causes relatively large astigmatism. Some lasers classified as index-guided also have weak evanescent waves. As a result, the beam waist in the horizontal direction is situated inwardly by ${ }_{\mathrm{L}}$ from the plane of beam emergence and thereby produces astigmatism. Whereas this astigmatism ${ }_{L}$ is as large as $10-50 \mu \mathrm{m}$ in the gain-guided laser, it is about $5-10 \mu \mathrm{m}(0.2-0.4 \mathrm{mil})$ in the index-guided laser. Figure 14.22 shows a typical distribution of astigmatism in index-guided lasers. Generally speaking, the astigmatism of these lasers tends to decrease as the laser output increases.

\subsubsection{Laser Noise ${ }^{18}$}

Semiconductor diode lasers heat up during operation because of power dissipation arising from the injection current. The temperature increase induces mode hopping, which is a small shift in the wavelength of the output light. Figure 14.23 shows the temperature
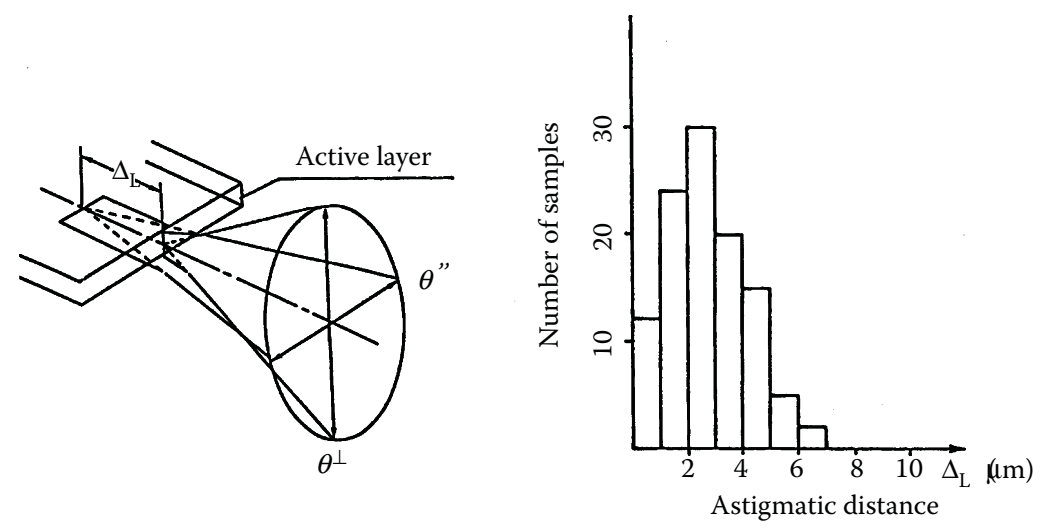

FIGURE 14.22

Distributions of astigmatic distance.

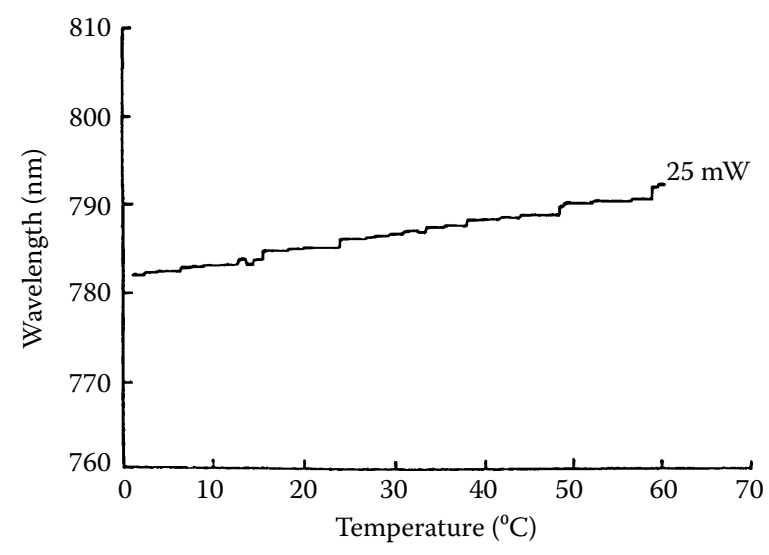

FIGURE 14.23

Temperature dependency of wavelength. 
dependence of a semiconductor laser. As the temperature of the laser increases, the longitudinal mode of the laser is shifted toward longer wavelengths. Substantial noise accompanies this type of mode hopping. Figure 14.24 shows the relative intensity of noise (RIN) versus the laser heat sink temperature. Figure 14.24a shows the characteristic of the element itself, and the broken line represents the allowable noise level for an optical pick-up.

If a small amount of the output beam is directed back into the output aperture of the laser, the laser emission will become unstable, exhibiting both mode hopping and excessive amplitude noise. If the level of return light is about $0.5 \%$, the relative intensity noise will be above the noise level allowable for an optical pick-up. Noise of this magnitude not only leads to a decrease in disk recording/reproduction SNR, but may also lead to instabilities in the focus and tracking servos.

An optical isolator consisting of a $\lambda / 4$ plate and a PBS is typically used in the optical path to minimize light reflections back into the laser output operations. However, the return light cannot be completely eliminated due to the birefringence in the disk and variations in isolator performance. As a consequence, the generation of noise caused by return light is inevitable in semiconductor lasers of longitudinal single mode. Within the constraints of the basic aspects of the optical pick-up design, return light noise can be best controlled by broadening the emission spectral line width of the semiconductor laser to reduce the coherence of the light. Introducing the multiple longitudinal modes can broaden the emission spectral line width.

In index-guided lasers, the transverse mode behavior becomes single mode at an emission output of $1 \mathrm{~mW}$ or higher, due to a confining effect on the transverse mode. In gainguided lasers, the transverse mode is confined by the gain corresponding to the carrier density and that generally results in multiple mode output. The influence of return light in a multimode laser is small and hence laser emission is unaffected. However, the inherent
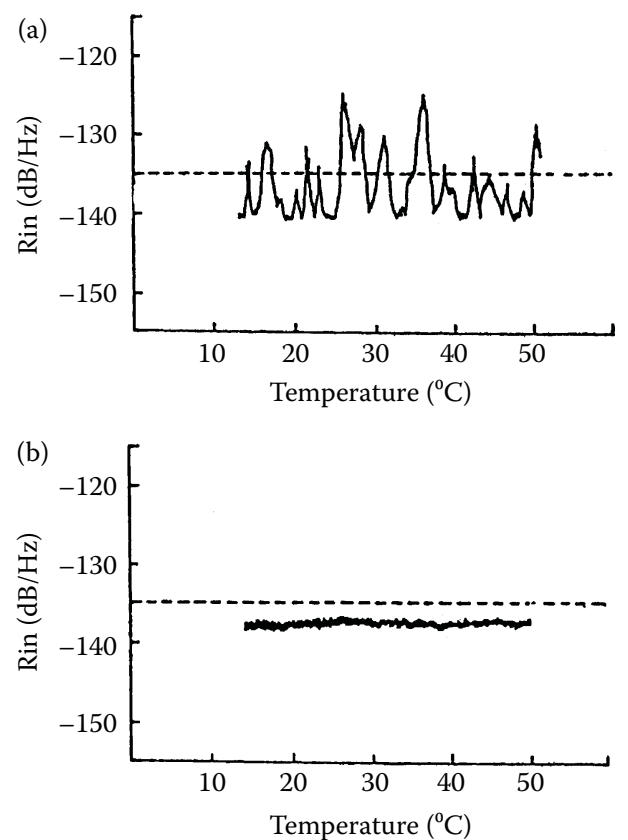

FIGURE 14.24

Noise characteristics of semiconductor laser: (a) inherent noise, (b) noise when modulated with high-frequency carrier. 
noise level is higher than in single-mode lasers. As shown in Figure 14.24b, when the indexguided laser operating single mode is modulated with high-frequency carrier, the longitudinal mode becomes multimode with the result that the noise level is lowered. Figure 14.25 shows the return-light noise levels of various lasers. Noise level RIN is calculated as:

$$
\operatorname{RIN}=\frac{\left\langle\Delta P^{2}\right\rangle}{P^{2} \Delta f}
$$

where $\left\langle\Delta P^{2}\right\rangle$ is the mean square of noise power, $P$ is the output power, and $f$ is the noise bandwidth. When the high-frequency oscillation is set at 300-600 MHz and the modulation level is set below the laser emission threshold, the light output becomes a pulse emission providing a multimode operation. Figure 14.26 shows an example of (upper curve) the read signal obtained without high-frequency oscillation and (lower curve) the read signal with high-frequency oscillation. The addition of the high-frequency oscillation improves the CNR of the carrier signal by about $5 \mathrm{~dB}$.

\subsection{FOCUSING AND TRACKING TECHNIQUES}

\subsubsection{Focusing Servo System and Method of Error Signal Detection}

The laser beam in an optical disk system is focused on the disk surface while the disk rotates at a high speed. An optical disk spinning at a high speed typically exhibits motion in the axial direction of tens to hundreds of micrometers. It is necessary that the OB follows this motion to keep the focus position of the beam on the signal plane of the disk

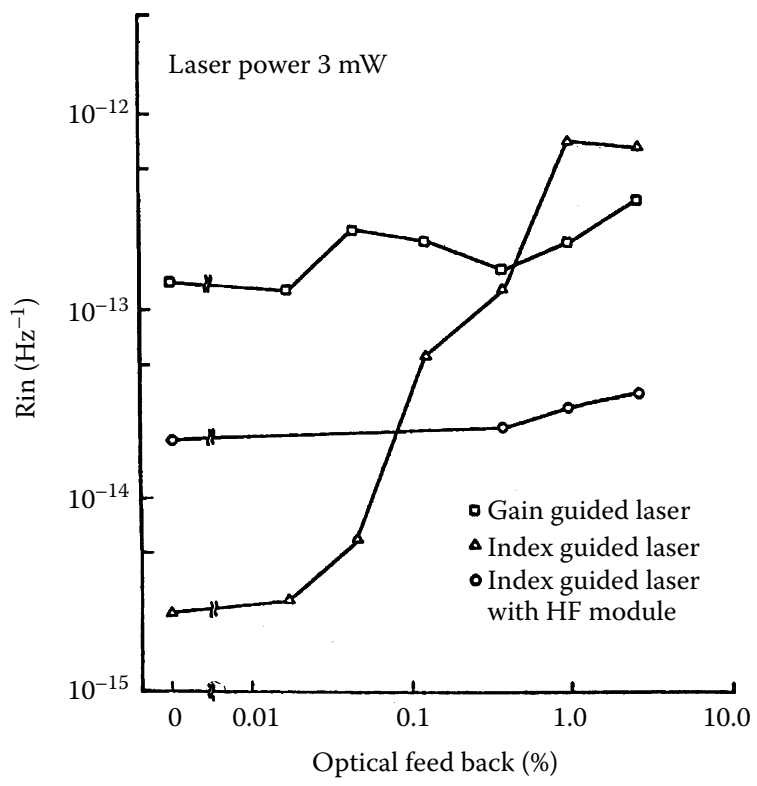

FIGURE 14.25

Laser noise versus optical feedback. 


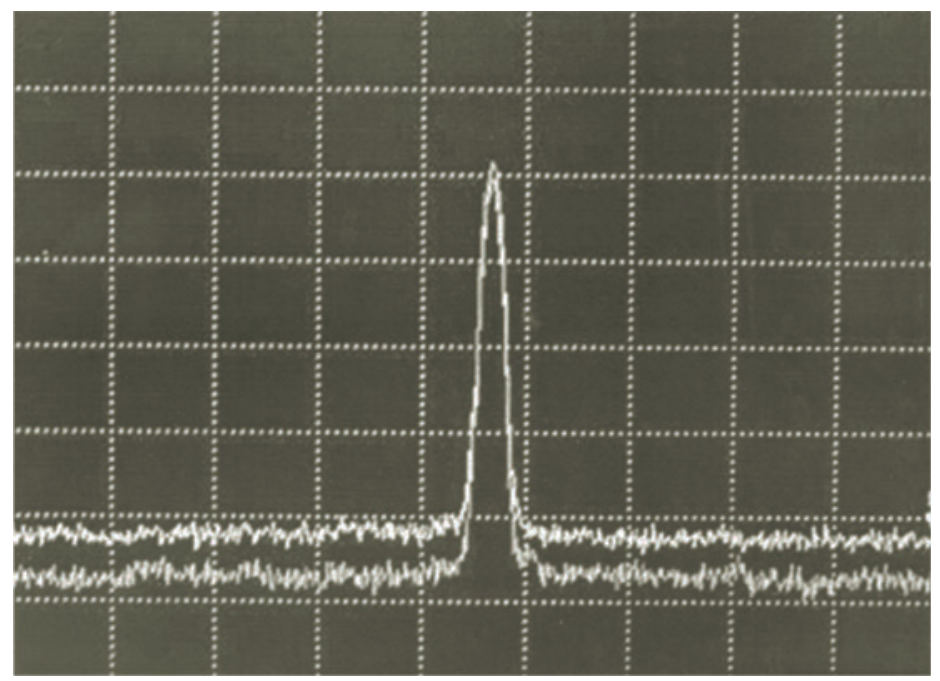

FIGURE 14.26

Reproducing carrier signals: upper baseline shows the noise level without high-frequency oscillation; lower baseline shows the noise level with high-frequency oscillation.

within the allowable limits of defocus of the optical system. The focusing mechanism generally used for this purpose is a moving-coil actuator employing a magnet and a coil. The required frequency response of the system is from several $\mathrm{Hz}$ to more than $10 \mathrm{kHz}$. Figure 14.27 shows a block diagram of the optical disk focusing system. The focusing servo loop comprises a focusing error signal detection unit, a circuit for amplification with phase correction of the detected error signal, and an actuator for driving the OB. This actuator is designed to follow the axial motion of the disk in response to a servo signal in the presence of external noise associated with the movement of the actuator and the interference from the tracking signal. In designing an autofocusing mechanism for the optical disk, external light noise must be reduced as much as possible. A balanced design must be employed and take into consideration: (1) interference from the tracking signal that occurs when the beam traverses the tracks; (2) mutual interference from motion of the focusing and tracking actuators; and (3) false focusing error signals associated with movement of the beam on the detector in the course of tracking.

Focusing errors are introduced by the axial motion of the disk, vibrations of the device and other causes. The focusing error information contained in the laser light reflected from the disk can be transformed into intensity or phase differences to derive an error signal. Each of the following beam characteristics can be utilized to generate a focusing error signal:

1. Change of beam shape

2. Movement of the beam position

3. Phase of the modulated waveform of the beam

\subsubsection{Beam Shape Detection Method}

Two separate techniques can be used to detect the beam shape to obtain a focusing error signal. These are the astigmatic focusing detection method and the spot size detection method. 
Figure 14.28 shows a basic optical system for the astigmatic focusing detection method using a tilted parallel plate. Although the conventional implementation includes use of a cylindrical lens, the tilted plate method is advantageous in the simplicity of the optics. The sensitivity of focusing error signal detection is dependent on the thickness and refractive index of the parallel plate assuming that the magnification of the OB is constant. The greater the thickness of the plate and/or the larger the refractive index, the larger the astigmatism and, hence, detection sensitivity. Figure 14.29 shows a typical focusing error signal in the optimum design. When the detection sensitivity is relatively low, defocus becomes large due to false signals caused by dropouts in the disk or movement of the OB during tracking. When the detection sensitivity is too high, the dynamic range of the focusing servo is diminished and the stability of the servo is decreased.

\subsubsection{Spot Size Detection Method}

Figure 14.30 shows the operating principle of the spot size detection method. The central part of the beam is received in front of and beyond the focal point of the beam by two three-segment detectors. A focusing error signal is derived from the intensity difference on the central and outer segments. The beam shape detection method generally has a large allowance for detector offset and has good temperature characteristics and aging stability. Recent progress of holographic technology enables use of a holographic optical element (HOE) to detect focusing signals. ${ }^{13}$

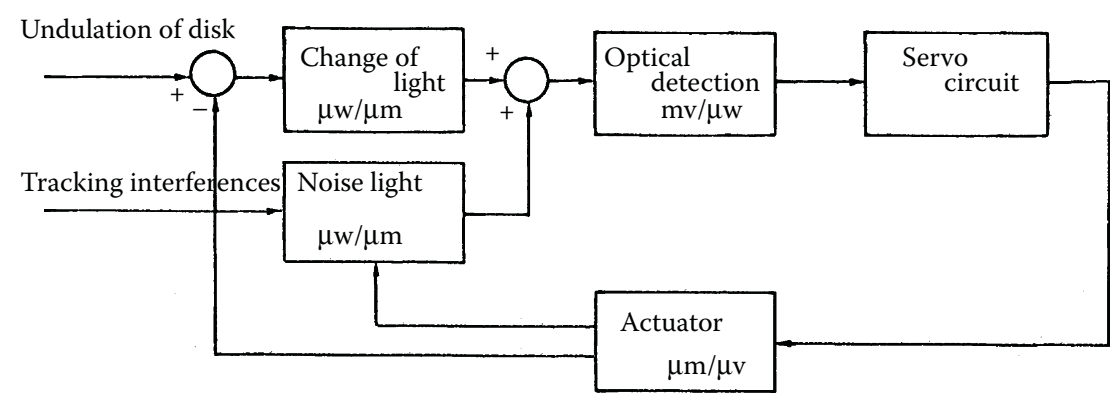

FIGURE 14.27

Block diagram of focusing servo.

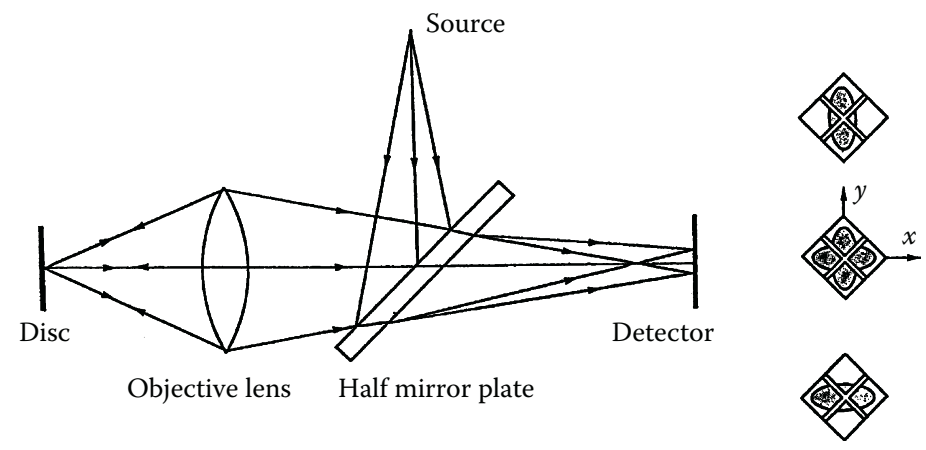

FIGURE 14.28

Astigmatic focusing method with plate. 


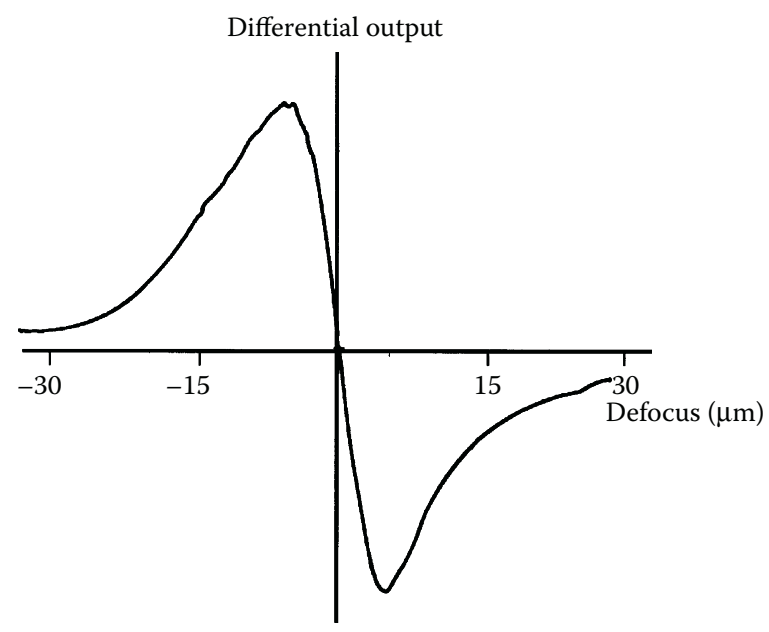

FIGURE 14.29

Typical astigmatic focusing error signal.

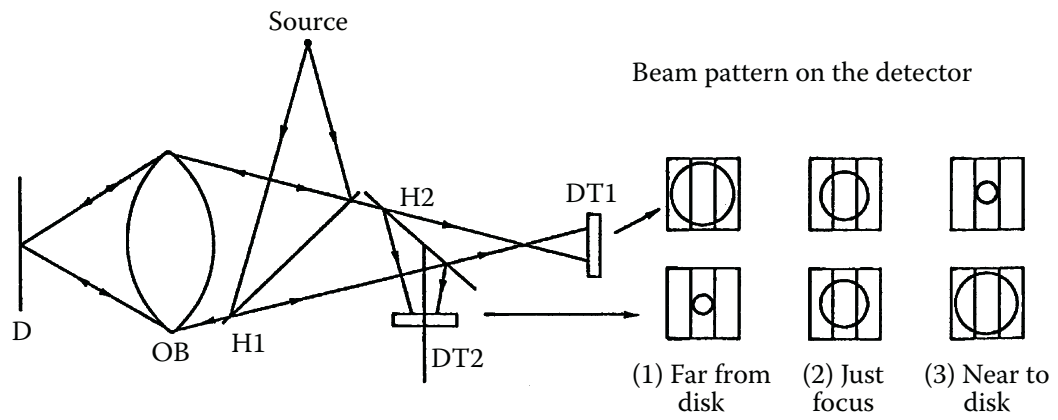

FIGURE 14.30

Spot size detection method.

\subsubsection{Beam Position Detection Method}

The beam position detection method converts the movement parallel to the optical axissuch as axial motion of the optical disk-to a beam movement in a plane perpendicular to the optical axis to obtain a focusing error signal. This detection method uses relatively simple hardware construction for detection and gives a broad focusing dynamic range.

Figure 14.31 shows a focusing error signal detection system using a bi-prism. This is an example of the Foucault focusing detection method. As the distance between the disk and the OB decreases, the intensity on the inner side of the respective split detectors increases and an increasing distance between the disk and the $\mathrm{OB}$ results in increasing intensity on the outer sides of the split detectors.

Figure 14.32 shows a focusing detection system using the critical angle of a prism. The beam of rays reflected from the disk enters the prism as a divergent beam when the distance between the disk and the OB is small, or as a convergent beam when the distance is large. When the prism is set at the critical angle, and the rays are not parallel, the beam will be partially transmitted by the prism to establish differential intensities on the 


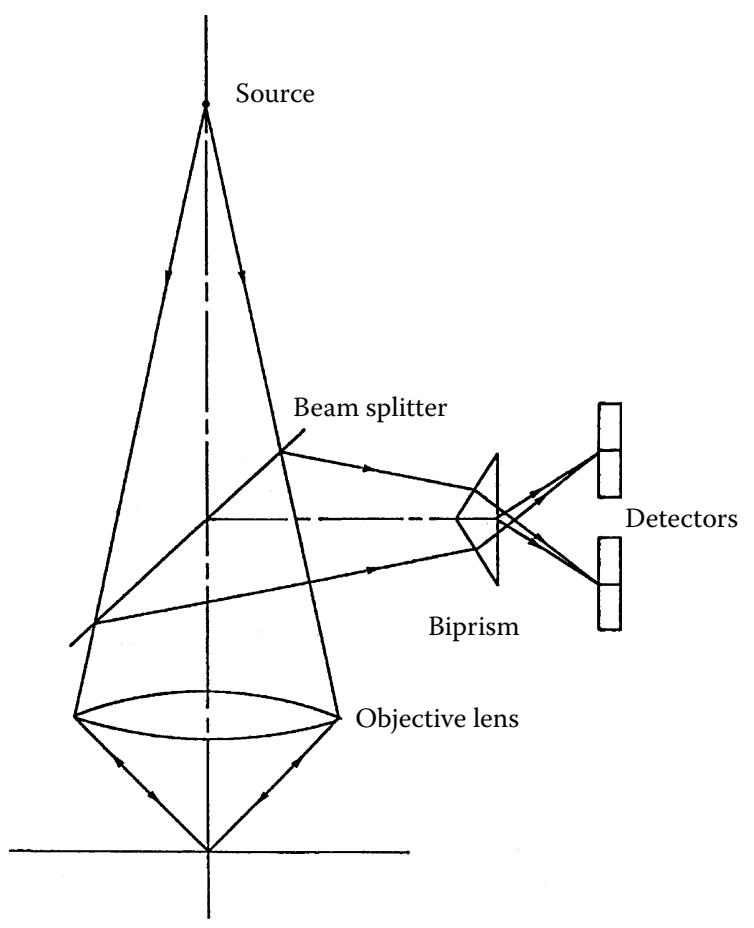

\section{FIGURE 14.31}

Foucault focusing method.

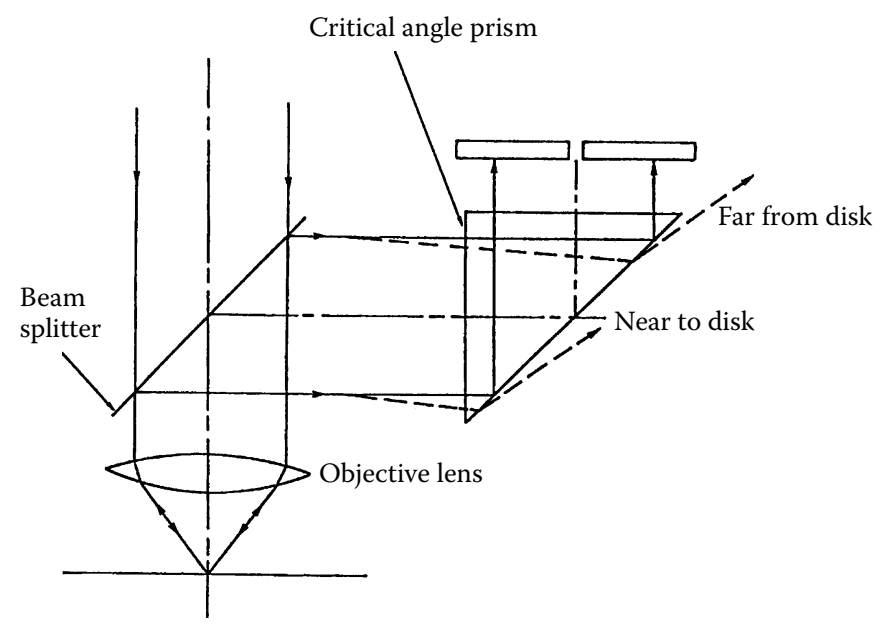

FIGURE 14.32

Critical angle focusing method.

detectors. In the case of a divergent beam, the near detector receives less light; in the case of a convergent beam, the far detector receives less light.

There are other methods for focusing detection, such as a skew beam focusing detection system, a system wherein the incident beam is eccentric with respect to the OB axis, a system using a single knife-edge, a beam rotation focusing detection system, and others. 


\subsubsection{Beam Phase Difference Detection}

There are two methods to detect beam phase difference: the spatial phase difference detection method and the temporal phase difference detection method. In the spatial phase difference detection method, illustrated in Figure 14.33, the phase of the beam located in the far-field pattern of the reflected beam diffracted by a given pattern in the optical disk (e.g., the pregrooved track pattern) is detected. This method is dependent on beam wavelength, and the dynamic range of focusing error signal is narrow. The temporal phase difference detection method is also known as the wobbling method. In this method, the focal point of the beam irradiating the optical disk is modulated along the optical axis with a wobbler. The phase of the modulated signal obtained with a detector is compared with the phase of the modulated drive signal of the wobbler to obtain a focusing error signal proportional to the phase difference.

\subsubsection{Track Error Signal Detection Method}

\subsubsection{Detection Methods}

The signal tracks on a DVD disk have a pitch of $0.74 \mu \mathrm{m}$, and the signal tracks on a conventional $C D$ disk have a pitch of $1.6 \mu \mathrm{m}$. The beam spot for reproducing the signal must follow this track within an accuracy of $0.04-0.1 \mu \mathrm{m}$. This tracking performance is achieved by driving the $\mathrm{OB}$ in a lateral direction with a voice coil actuator. The following methods are commonly used for optical detection of the TE:

1. Detection using two auxiliary beams generated by grating (3-B or 3-beam method)

2. Detection of the far-field distribution of the read/write beam reflected from the disk (PP method)

3. Detection from the difference between two signal levels obtained with sample pits disposed at an offset of $\pm 1 / 4$ pitch from the track (SS or sampled servo method)

4. Detection of the phase difference between the differential output from diagonal playback signal of quadrant detector and the sum output signal of quadrant detector (DPD or differential phase detection method)

5. Detection of the phase difference between the playback signal obtained by a slight induced displacement of the beam in the direction perpendicular to the track and the phase of the corresponding drive signal (wobbling method)
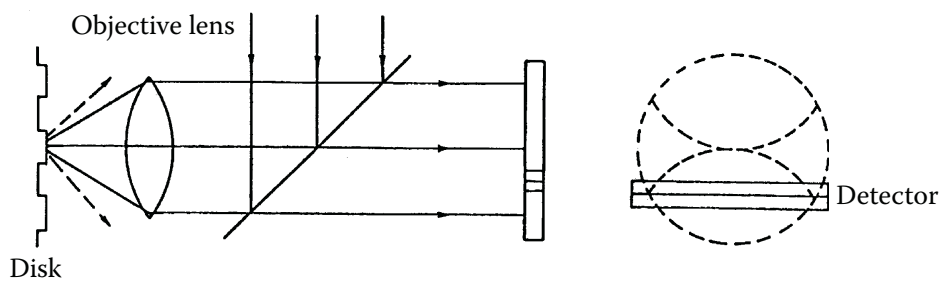

FIGURE 14.33

Spatial phase detection method. 


\subsubsection{3-Beam Method}

The 3-beam method using auxiliary beams is shown in Figure 14.34. The two first-order beams obtained by passing the laser beam through a diffractive grating are aligned to positions on the disk of about plus and minus one-quarter of the track pitch apart from the track center (B1, B2). The reflected two beams are received by two detectors (D1, D2) to obtain a track error signal. The fundamental beam (B0) is used for SG detection by a central detector (D0). While this method is well suited to the read-only optical pick-up like $C D$, it must be carefully designed for use in read/write optics. In this case the beam intensity is increased during the writing mode, introducing the risk of harmful recording by the auxiliary beams.

\subsubsection{Wobbling Method}

In the wobbling method, the track error signal corresponds to the phase difference between a signal to the transducer that induces a slight displacement of the beam in the direction perpendicular to the track and a signal from the beam modulated by track edge diffraction. This method has only been implemented in certain limited applications. This is partly due to the poor stability of the wobbling frequency and partly due to the $0.1 \mu \mathrm{m}$ wobble displacement required to obtain a TE with satisfactory $\mathrm{S} / \mathrm{N}$ ratio. Wobble displacement of $0.1 \mu \mathrm{m}$ is close to the maximum allowable tracking error value.

\subsubsection{Differential Phase Detection (DPD) Method}

This method uses the quadrant detectors to detect the phase difference between the differential output from the diagonal playback signal (D1 + D4) $-(\mathrm{D} 2+\mathrm{D} 3)$ of the quadrant detector and the sum output signal (D1 + D2 + D3 + D4) of the quadrant detector. The DPD tracking error detection is shown in Figure 14.35. This is the method recommended for playback TE detection in the DVD specifications. ${ }^{19}$
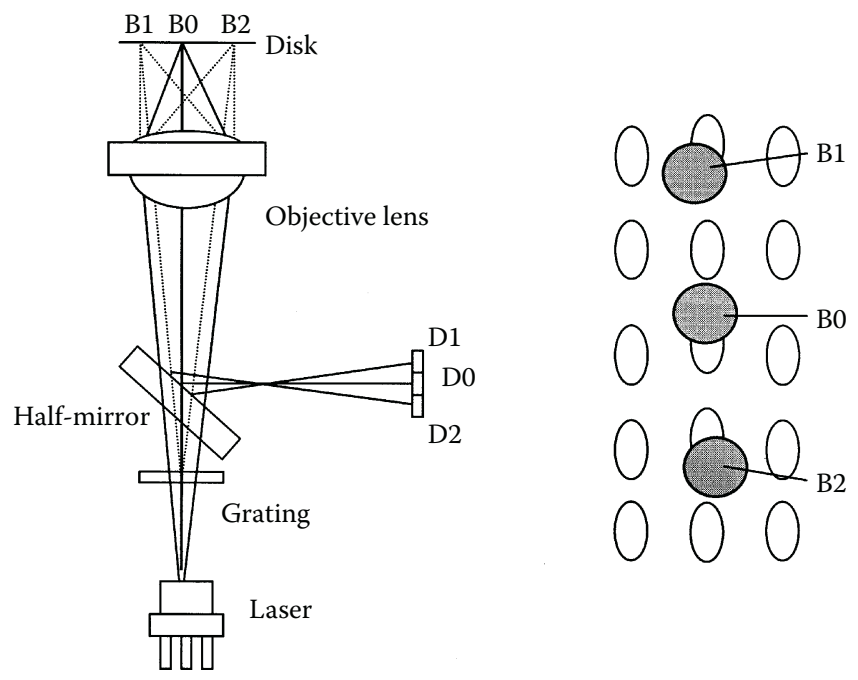

FIGURE 14.34

Tracking error signal detection with 3-beam method. 


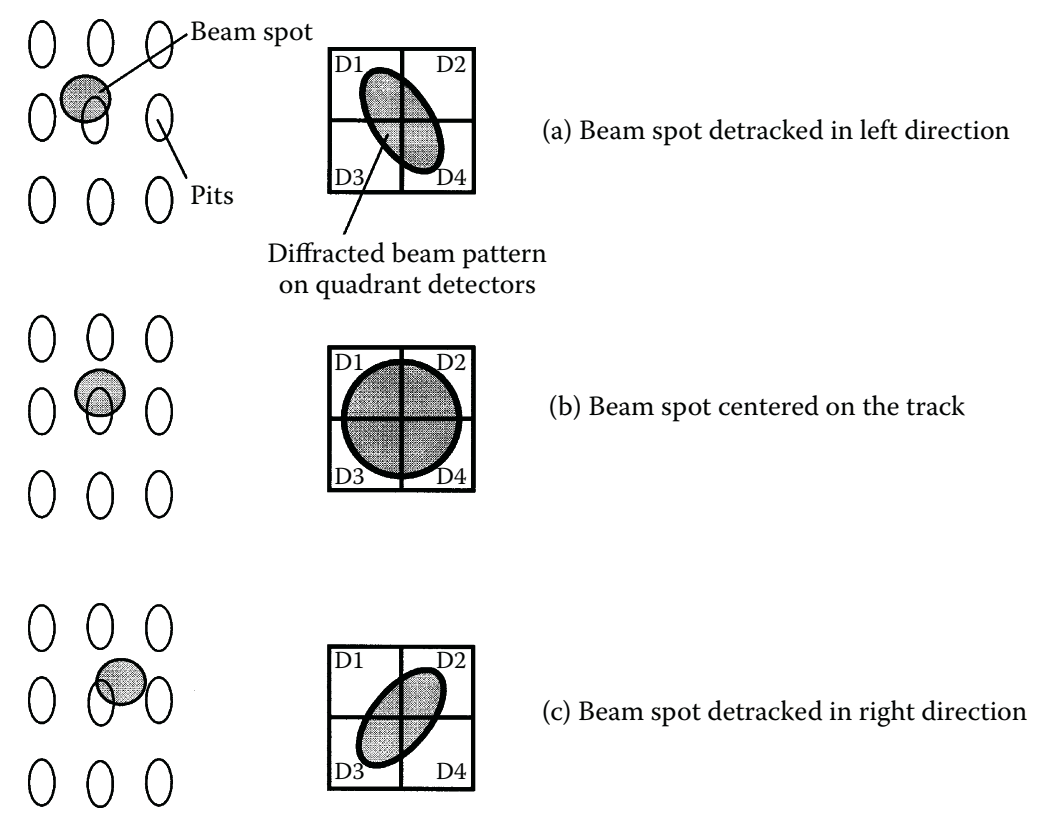

FIGURE 14.35

Differential phase detection method.

\subsubsection{Push-Pull Track Error Signal Detection Method}

The simplest method for obtaining a track error signal in read/write is called the "pushpull method." A split detector is inserted in the far-field of the beam in such a manner that the line of division of the detector is lined up with the track.

Figure 14.36 shows a basic optical system for signal detection using the PP method. The intensity pattern incident on the split detector is a combination of the zero-order and firstorder beams (due to diffraction caused by the track on the disk). The track error signal is derived from the difference in the signals from the two detectors. Figure 14.37 shows the far-field beam distributions according to the beam spot position on the track. The asymmetry of the far-field beam intensity distribution and the track error signal level are maximum when the track groove depth is $\lambda / 8$. (When the depth is $\lambda / 4$ multiplied by an integer, asymmetry disappears and no track error signal is obtained.)

\subsubsection{Slit Detection Method ${ }^{20}$}

When the beam spot is located in the center of the track, the phase difference $\psi$ between the zero-order beam and the two first-order beams in the PP system is dependent on the diffraction by the track and on the defocus $\Delta \mathrm{Z}$ and can be expressed as ${ }^{21}$

$$
y=\frac{p}{2}+\frac{2 p}{1}\left[\sqrt{1-\left(\frac{1}{p}-\sin a\right)^{2}}-\cos (a)\right] \Delta Z
$$

where $\alpha$ is an angle between the optical axis and an arbitrary point in a far-field image. 


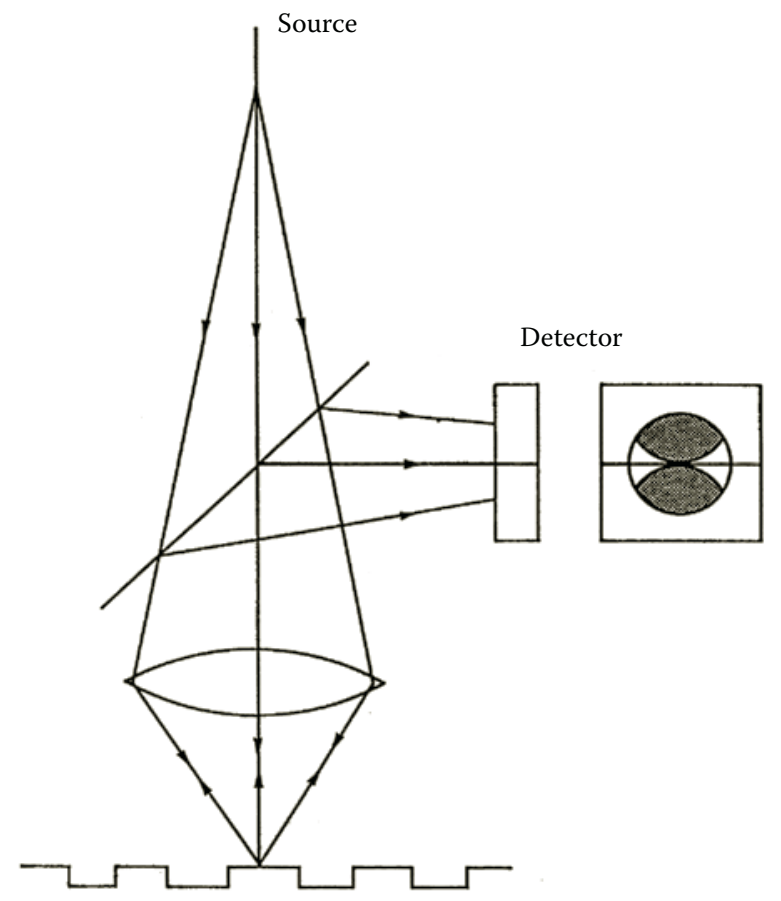

FIGURE 14.36

Push-pull method.

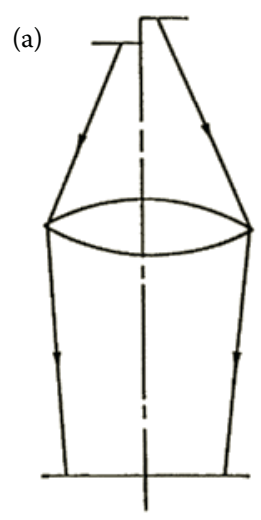

(b)
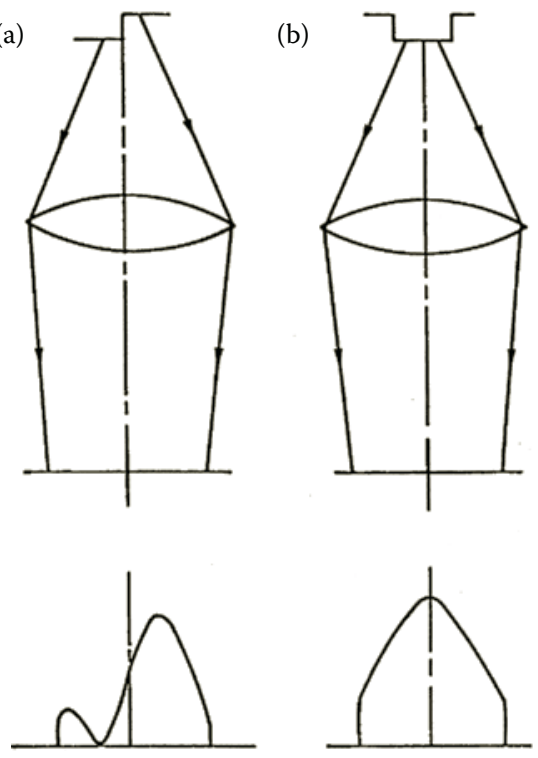

(c)
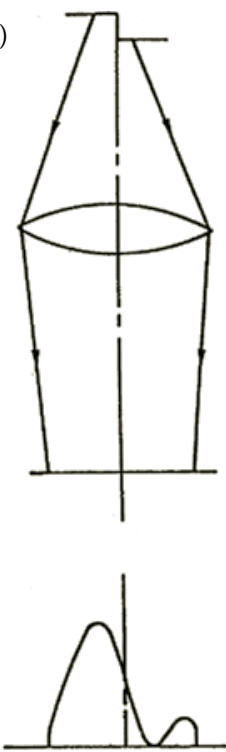

FIGURE 14.37

Far-field beam distribution at various locations on the track. 
From Equation 14.17, the phase difference is constant for $a=\sin ^{-1}(1 / 2 p)$ in the far-field image and independent of defocus but exclusively dependent on diffraction by the track. Figure 14.38 shows a typical far-field beam distribution in the presence of defocus. By utilizing this property in the far-field, the control range of tracking with respect to defocus can be expanded. Thus, the defocus characteristic of the track error signal can be improved by providing slits symmetrically in the centers of overlaps between the zero-order beam and the two first-order beams as illustrated in Figure 14.39.

Figure 14.40 shows the change in track error signal level according to defocus at various slit widths. If the slit width is about $20 \%$ of the far-field pattern, the proportion of change in the track error signal due to defocus is improved by about a factor of 2 . If the slit width

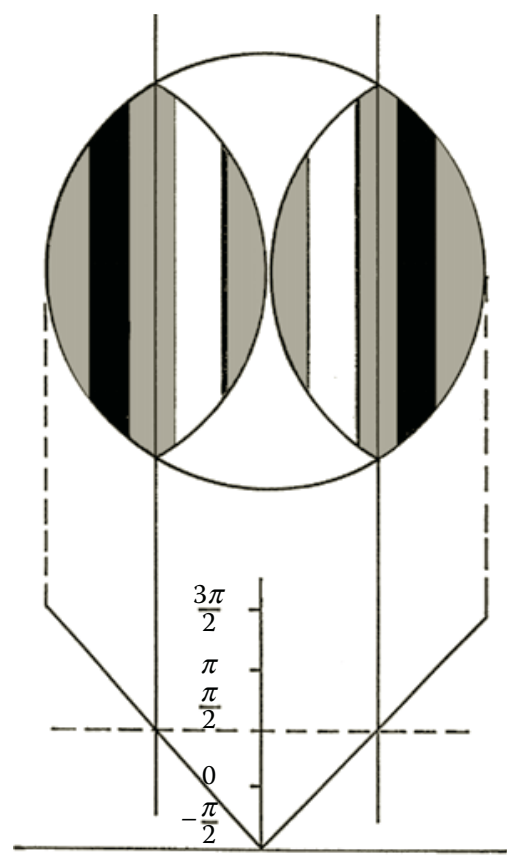

FIGURE 14.38

Far-field pattern when defocus occurred.

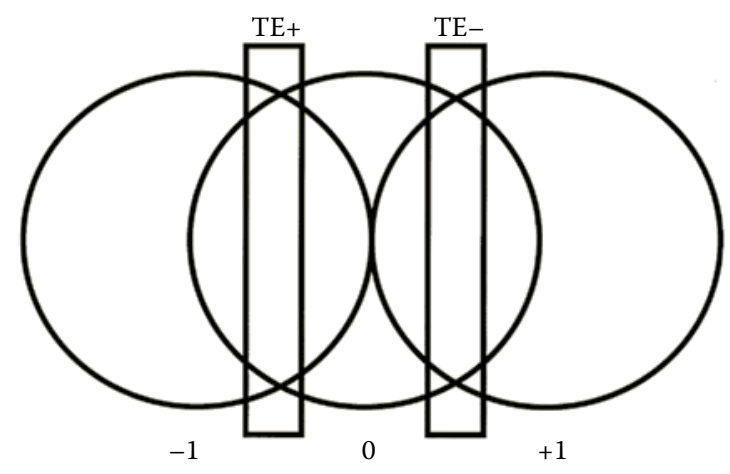

FIGURE 14.39

Slit detection method. 


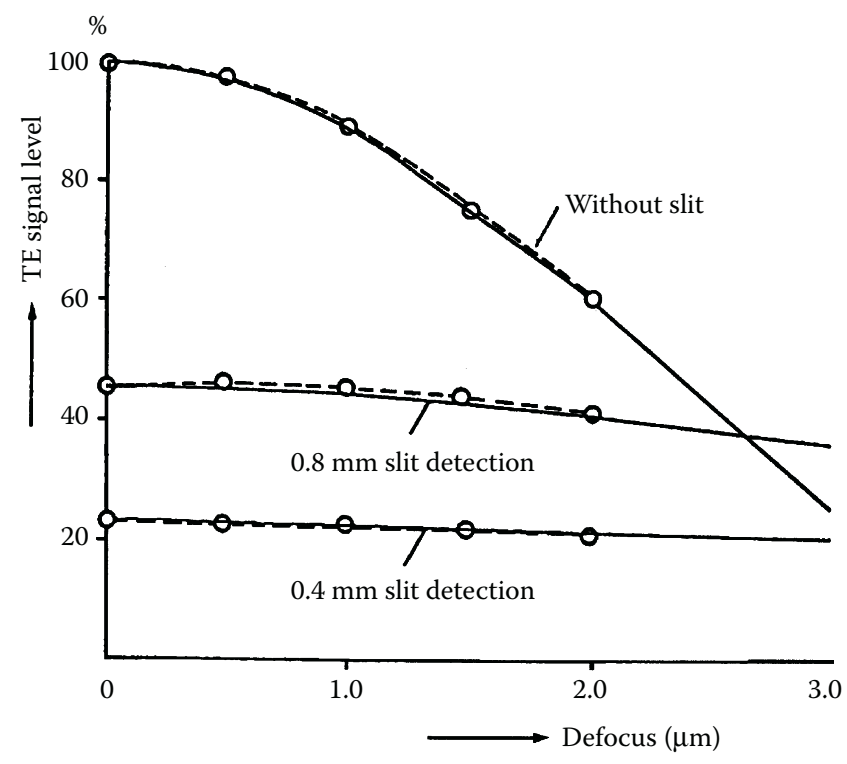

FIGURE 14.40

Defocus versus tracking error signal level.

is made too narrow, the $\mathrm{S} / \mathrm{N}$ of the track error signal will decrease. The optical parameters in these experiments and theoretical calculations are as follows:

1. Objective lens: $\mathrm{NA}=0.5$

2. Laser wavelength: $L=830 \mathrm{~nm}$

3. Track pitch: $t=1.6 \mu \mathrm{m}$

4. Slit width: $W=0.8$ and $0.4 \mathrm{~mm}$

\subsubsection{Sampled Tracking Method ${ }^{22}$}

In a sampled tracking system, track error signal detection pits are periodically provided in lieu of the continuous groove in a conventional grooved disk. The sample pits consist of two pits displaced by about $\pm 1 / 4$ of the track pitch from the track center and one pit centered on the track. Figure 14.41 shows the principle of sampled track error signal detection. When the beam spot is (a) off-track upwardly from the track center, the first pit output is large and the second pit output is small. In the on-track condition, the first pit output level and the second pit output level are equal (b). If the beam spot is downwardly off-track, the first pit output is small and the second pit output is large (c). The off-track condition is diagnosed by comparing these pit output levels. The third pit is used for making a sampling clock, and the track error signal detection is constructed from these outputs. Figure 14.42 shows a block diagram of the detection circuit.

In the sampled tracking system, each set of pits provided for the track error signal detection uses a track length equivalent to that used to store 1 byte of information. The sampling frequency must be higher than about ten times the cutoff frequency of the tracking servo. This reduces the size of the usable data area, but the overall system performance improves because there is less degradation of data signals and interference effects on the focusing 


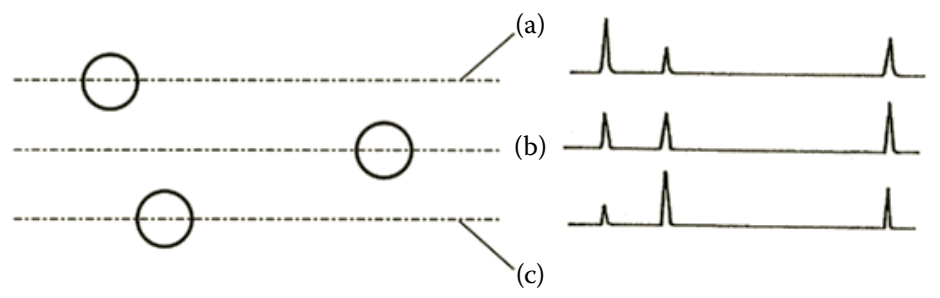

\section{FIGURE 14.41}

Tracking error signal detection from sampled pits: (a) off-track upwardly from the track center, (b) on-track, (c) off-track downwardly from the track-center.

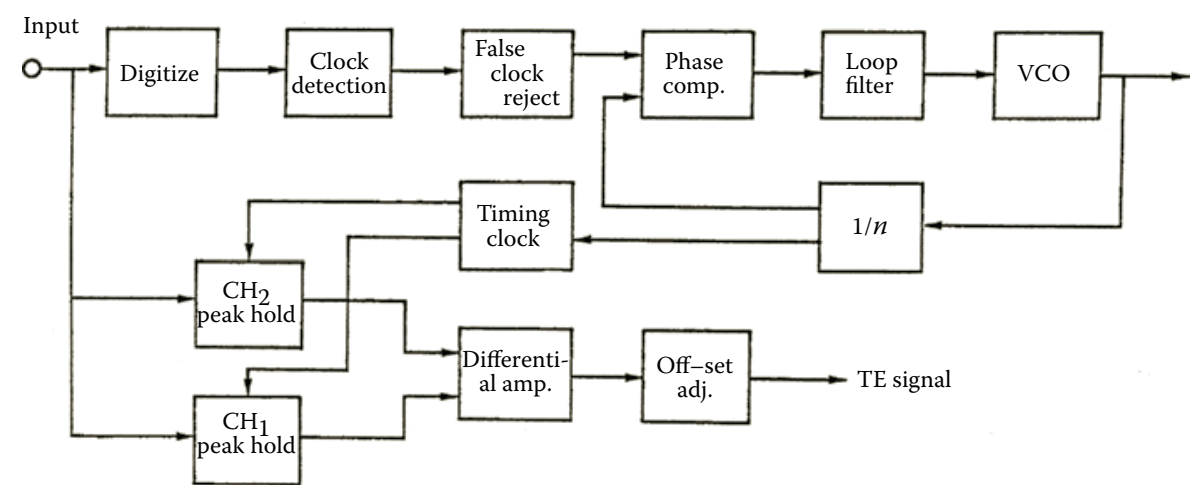

FIGURE 14.42

Block diagram of sampled servo tracking method.

servo caused by the track groove. Further, an inclination of the disk induces a track offset in the PP system, but not in the sampled tracking method. For example, a disk inclination of $0.7^{\circ}$ of a $1.2-\mathrm{mm}$-thick substrate with $\mathrm{NA}=0.5$ and $\lambda=830 \mathrm{~nm}$ causes a $0.1 \mu \mathrm{m}$ lateral shift in the quiescent operating position of the PP tracking servo. This type of systematic track error is decreased by about a factor of 5 when the sampled tracking method is used.

\subsection{RADIAL ACCESS AND DRIVING TECHNIQUE}

\subsubsection{Fast Random Access}

A critical aspect of an optical disk memory system is fast random access to the stored information. This random access is accomplished via two mechanisms: optical pick-up motion for rough positioning and the tracking actuator for precise positioning. A linear actuator is used as the coarse positioning means. To minimize access time, it is necessary to (1) develop a small and lightweight optical pick-up; (2) increase the resonant frequency of the linear actuator; and (3) develop a transfer mechanism with a minimum of friction. In the typical linear actuator designed for video recording applications, the transfer segment weighs only $78 \mathrm{~g}$ and has a thrust of $3.0 \mathrm{~N} / \mathrm{A}$. This linear actuator gives an average access time of 75 ms or less. ${ }^{9}$ The optical pick-up base is provided with roller bearings so that it 
freely moves on the guide rods. The low-frequency component of the track error signal is fed to the linear actuator so that the center of drive of the OB will lie at the center of the tracking mechanism motion range.

Figure 14.43 shows the modes of access by the linear actuator/tracking actuator combination ${ }^{23}$ First, the tracking actuator is disabled. Then the linear actuator is accelerated and decelerated at the maximum speed to position the optical pick-up in the vicinity of the correct track (A to B). The tracking actuator is reactivated and the track address is read (B to $\mathrm{C}$ ). The number of tracks between the actual and desired address is calculated, and access is completed by executing a multitrack jump. Figure 14.44 is a block diagram of the tracking servo circuit and linear actuator circuitry. The track error signal obtained by the procedure described in Sec. 5.2 is fed to an amplification circuit, a switching circuit, and a drive circuit in succession to drive the tracking actuator. The tracking drive signal is also used to drive the linear actuator. During random access, the drive signal is removed from

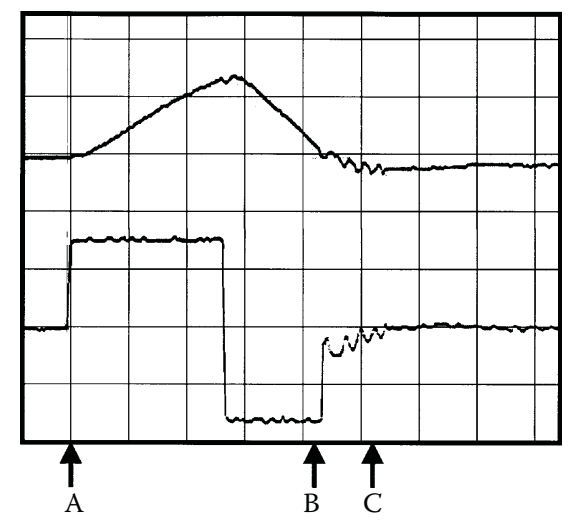

FIGURE 14.43

Modes of access by the linear actuator and tracking actuator.

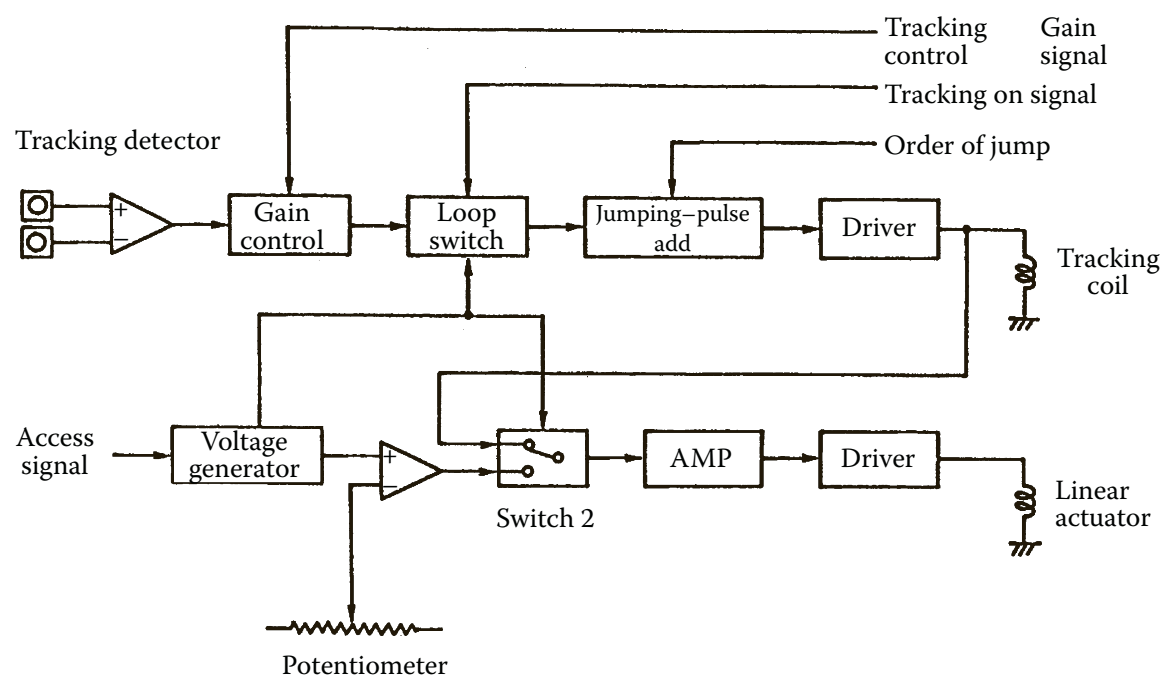

FIGURE 14.44

Block diagram of tracking and accessing servo. 
the tracking actuator and a voltage corresponding to the access signal is generated and supplied to the linear actuator drive circuit. Two methods are available for ascertaining the actual position with respect to the target track. The first method involves calculating the number of tracks between the current and desired positions, then detecting and counting optically each pregroove as it is passed over in the radial scan. The scan is stopped when the correct number of tracks has been crossed.

Since this method counts the tracks themselves, the distance to the target track can be accurately computed. The track detection bandwidth must be broad enough to prevent miscounts of the tracks during the peak speed of the linear actuator. This method is less applicable when using sampled format disks; even track addresses can complicate the track-counting process in continuous format disks. A second method provides the optical pick-up with a position sensor for detecting the current position. This provides a stable position signal, and the access servo can be damped using the output signal from this sensor. Examples of position sensors include linear scale sensors, optical position sensors, and slide resistance sensors. Figure 14.45 shows a typical optical position sensor.

\subsubsection{Optical Drive System}

The optical disk system consists of hardware comprising the disk, optical pick-up, accessing circuit, signal processing circuit, error correction circuit, microcomputer, and so on, and software for processing the various signals. The height of the 5.25 in optical disk drive is either full height $(82 \mathrm{~mm})$, half-height $(41 \mathrm{~mm}), 1$ in height, or half-inch height, corresponding to standardized magnetic disk products. In the half-height drive, design goals include the use of a disk cartridge that is inserted and clamped and low profiles of the component parts for the access mechanism. The height of the optical pick-up must be less than 15-16 mm. For the thinner drive like those used in a notebook computer, the height of the drive is less than $12.7 \mathrm{~mm}$. A height of the optical pick-up of around $7.5 \mathrm{~mm}$ is needed

(a)

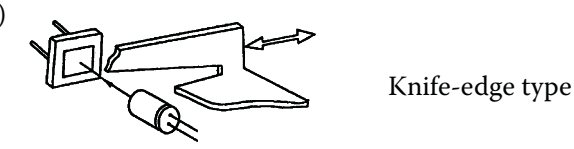

(b)

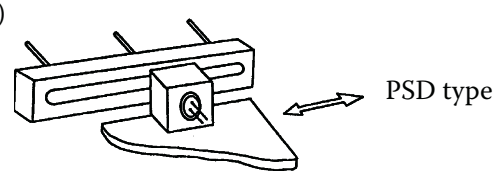

FIGURE 14.45

Optical position sensor: (a) knife-edge type; (b) PSD type.

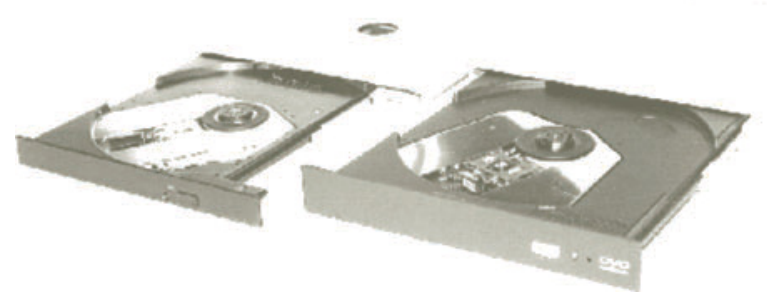

FIGURE 14.46

Thin optical disk drive for DVD-ROM. 
and an ingenious design is required. Figure 14.46 shows the thin optical disk drive for a DVD-ROM. The removability of the optical disk affects differences in the amounts of eccentricity and undulation each time a disk is mounted. Moreover, since the disk substrate is made of plastic, the amount of undulation increases with the age of the disk. The dynamic balance of the disk is affected by these factors, and vibrations are induced. It is necessary, therefore, to design the various actuators such that these vibrations do not degrade performance.

\section{ACKNOWLEDGMENTS}

I give my special thanks to Gerald F. Marshall, volume editor of Handbook of Optical and Laser Scanning, who has given me a chance to contribute to this book and is generous over my untrained English. I extend my profound thanks to the two following reviewers of my chapter, experts of optics, for their patient work in pointing out important directions and suggestions: Masud Mansuripur of the University of Arizona, and David Strand, of Energy Conversion Devices, Inc.

\section{Appendix A}

When the amplitude distribution in the pupil is given by $f\left(r^{2}\right)$ and the radius of the pupil is one unit, the integral of Fourier-Bessel transform is written as

$$
g(s)=\int_{0}^{1} f\left(r^{2}\right) J_{0}(s r) \mathrm{d}\left(r^{2}\right)
$$

A plurality of solutions exist for this integral. However, the solution given by A. Boivin ${ }^{24}$ is easily understood. Thus, the amplitude of the Fourier spectrum $g(w)$ is written in the form of a Bessel series:

$$
g(s)=\sum(-1)^{n} 2^{n+1} f_{(1)}^{n} \frac{J_{n+1}(s)}{s^{n+1}}
$$

where $f^{n}\left(r^{2}\right)$ denotes the $n$th differential of the function $f\left(r^{2}\right)$. For calculating the Fourier spectrum of a truncated Gaussian, $f\left(r^{2}\right)$ is expressed by exp $\left(-a r^{2}\right)$. Then the integral of Fourier-Bessel transform is written as

$$
g(s)=\int_{0}^{1} \exp \left(-a r^{2}\right) J_{0}(s r) r \mathrm{~d} r
$$

and the result becomes ${ }^{24}$

$$
g(s)=\sum_{n=0}^{\infty} 2^{n} a^{n} e^{-a}\left[\frac{2 J_{n+1}(s)}{s^{n+1}}\right]
$$




\section{Appendix B}

Thickness variations, index changes, and tilts of the disk substrate all cause wavefront aberrations. Here, we calculate the optical path differences $\Delta_{0}$ between two rays: the first is the on-axis ray, and the second is the outermost ray, which determines the NA of the OB. From Figure 14.47, the following relations can be easily calculated:

$$
\begin{gathered}
\sin (y-q)=n \sin \left(r_{1}\right) \\
\sin (q)=n \sin \left(r_{0}\right) \\
\Delta_{0}=n t\left\{1 / \cos \left(r_{1}\right)-1 / \cos \left(r_{0}\right)\right\}+t\left\{\cos (y-q) /\left[\cos \left(r_{1}\right) \cos (q)\right]-1 / \cos \left(r_{1}\right)\right\} / n
\end{gathered}
$$

Developing the power series of $\psi$ and $\theta$, the next quadratic terms are obtained:

$$
\Delta=t(1-n)^{2}\left\{y^{4}-4 y^{3} q+8 y^{2} q^{2}+8 y q^{3}\right\} / 8 n^{3}
$$

where $\psi$ is the NA of the OB and $t$ is the thickness of the disk substrate. Here, each term denotes the Siedel aberration. When only a thickness error $\Delta t$ exists, the spherical aberration $S_{1}$ is generated:

$$
S_{1}=\frac{\left(n^{2}-1\right) y^{4} \Delta t}{8 n^{3}}
$$

The relation between the wave aberration $W_{\mathrm{ST}}$ and the spherical aberration $S_{1}$ can be calculated from Maréchal's equation: ${ }^{25}$

$$
W_{\mathrm{ST}}^{2}=\frac{d^{2}}{12}+\frac{d S_{1}}{6}+\frac{4 S_{1}^{2}}{45}=\frac{\left(d+S_{1}\right)^{2}}{12}+\frac{S_{1}^{2}}{180}
$$

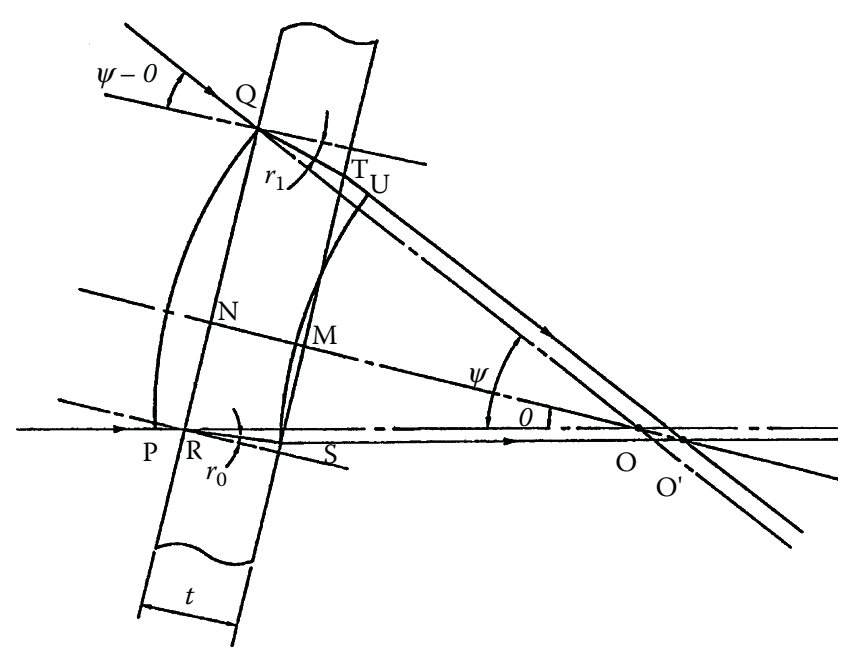

FIGURE 14.47

Optical path differences between two rays. 
where the wave aberration $W_{\mathrm{ST}}$ is minimal when the defocus $d$ is equal to the third spherical aberration $S_{1}$. Thus, the wave aberration due to the spherical aberration becomes

$$
W_{\mathrm{ST}}=\frac{S_{1}}{\sqrt{180}}=\frac{\Delta t\left(n^{2}-1\right)(N A)^{4}}{8 \sqrt{180} n^{3}}
$$

For a small tilt $\theta$ of the disk substrate, high orders can be disregarded and the only important aberration is the coma $C_{1}$ :

$$
C_{1}=t\left(n^{2}-1\right) y^{3} q / 2 n^{3}
$$

The relationship between the wave aberration $W_{\mathrm{TL}}$ and the coma $C_{1}$ can be calculated, again from Maréchal's equation ${ }^{25}$

$$
W_{\mathrm{TL}}^{2}=K^{2} / 12-K C_{1} / 6+C_{1}^{2} / 18=\left(K-C_{1}\right)^{2} / 12+C_{1}^{2} / 72
$$

where the wave aberration $W_{\mathrm{TL}}$ is minimal when the tilt of wavefront $K$ is equal to coma $C_{1}$. Then the wave aberration $W_{\mathrm{TL}}$ due to a tilt of the disk substrate becomes

$$
W_{\mathrm{TL}}=\frac{C_{1}}{\sqrt{72}}=\frac{t\left(n^{2}-1\right) y^{3} q}{2 \sqrt{72} n^{3}}
$$

\section{Appendix C}

The allowable limit of laser-mounting angle is calculated. When the wavefront of the beam emerging from a laser is astigmatic and its axis is not in agreement with the $x, y$-axis of the optics, there exists a residual astigmatism. With the y-axis as a reference, the phase difference $\psi(x)$ along the $x$-axis within the pupil plane of the CL with an astigmatic distance of $\Delta_{\mathrm{L}}$ and a focal length of $f_{\mathrm{c}}$ is expressed by

$$
y(x)=\Delta_{\mathrm{L}} x^{2} /\left(2 f_{\mathrm{c}}^{2}\right)
$$

Assuming that the wavefront is inclined through an angle $\theta$ with respect to the $y$-axis, the phase difference $\psi(x, y)$ is expressed as

$$
\begin{aligned}
y(x, y) & =\Delta_{\mathrm{L}}(x-y \tan q)^{2} /\left(2 \cos ^{2} q f_{\mathrm{c}}^{2}\right) \\
& =\Delta_{\mathrm{L}}\left(x^{2}+y^{2} \tan ^{2} q-2 x y \tan q\right) /\left(2 \cos ^{2} q f_{\mathrm{c}}^{2}\right)
\end{aligned}
$$

By focusing the optics, the term $x^{2}+y^{2} \tan ^{2}(\theta)$ can be zero. Therefore, the wavefront aberration assumes a maximum value in the direction of $x=y=h$ :

$$
y_{\mathrm{o}}=\Delta_{\mathrm{L}} \tan q h^{2} /\left(\cos ^{2} q f_{\mathrm{c}}^{2}\right)
$$


Since $h / f_{c}$ is the NA of the CL, the above equation may be rewritten as:

$$
y_{\mathrm{o}}=\Delta_{\mathrm{L}} \tan q\left(\mathrm{NA}_{\mathrm{c}}\right)^{2} / \cos ^{2} q
$$

Since Maréchal's equation gives the relationship between maximum astigmatism $\psi_{o}$ and wavefront aberration $W_{\mathrm{LA}}$ as

$$
W_{\mathrm{LA}}^{2}=\frac{y_{\mathrm{o}}^{2}}{6}
$$

we obtain

$$
W_{\mathrm{LA}}=\frac{\Delta_{\mathrm{L}} \tan q\left(\mathrm{NA}_{\mathrm{c}}\right)^{2}}{\sqrt{6} \cos ^{2} q}
$$

\section{REFERENCES}

1. Feinleib, J.; de Neufville, J.; Moss, S.C; Ovshinsky, S.R. Rapid reversible light-induced crystallization of amorphous semiconductors. Appl. Phys. Lett. 1971, 18, 254.

2. Hopkins, H.H. Diffraction theory of laser readout systems for optical video disks. J. Opt. Soc. Am. 1979, 69, 4-24.

3. Goodman, J.W. Introduction to Fourier Optics; McGraw Hill: New York, 1968; Chap. 6.3.

4. Braat, J. Principles of Optical Disk System; Adam Hilger Ltd.: New York, 1985; 7-85.

5. Firester, A.H.; Caroll, C.B.; Gorog, I.; Heller, M.E.; Russell, J.P.; Stewart, W.C. Optical read out of RCA video disk. RCA Review 1978, 39(3), 392-407.

6. Mansuripur, M. Scanning optical microscopy part 1. Opt. \& Photonics News 1998, May, 56-59.

7. Yoshida, T. Tellurium sub-oxide thin film disk. Proc. SPIE Optical Disks Systems and Applications 1983, 421, 79-84.

8. Saimi, T. Compact optical pick-up for three dimensional recording and playing system. CLEO '82 Pheonix, April 1982.

9. Imanaka, R.; Saimi, T.; Okino, Y.; Tanji, T.; Yoshimatsu, T.; Yoshizumi, K.; Kamio, K. Recording and playing system having a compatibility with mass produced replica disk. IEEE Consumer Electonics 1983, CE-29(3), 135-140.

10. Hartmann, M.; Jacobs, B.A.J.; Braat, J.J.M. Erasable magneto-optical recording. Philips Tech. Rev. 1985, 42(2), 37-47.

11. Deguchi, T.; Katayama, H.; Takahashi, A.; Ohta, K.; Kobayashi, S.; Okamoto, T. Digital magneto-optical disk drive. Appl. Opt. 1984, 23(22), 3972-3978.

12. Born, M.; Wolf, E. Principles of Optics; Pergamon Press: Oxford, 1970.

13. Saimi, T. PD Head for "PD" System, National Technical Report, Dec. 1995; Vol. 41, No. 41.

14. Shih, Hsi-Fu. Holographic laser module with dual wavelength for digital versatile disk optical heads. Jpn. J. Appl. Phys. 1999, 38, 1750-1754.

15. Nakamura, H. Fine Focus 1-Beam Optical Pick-Up System, National Technical Report, 1986; 72-80.

16. Finck, J.C.J.; van der Laak, H.J.M.; Schrama, J.T. A semiconductor laser for information readout. Philips Tech. Rev. 1980, 139(2), 37-47.

17. Imafuji, O.; Fukuhisa, T.; Yuri, M.; Mannoh, M.; Yoshikawa, A.; Itoh, K. Low operating current and high-temperature operation of 650-nm AlGalnP high-power laser diode with real refractive index guided self-aligned structure. IEEE J. Selected Topics in Quantum Electronics 1999, 5(3), 721-728. 
18. Chinone, N.; Ojima, M.; Nakamura, M. A semiconductor laser below allowance of noise due to the optical feedback by adding the high frequency generating circuit. Nikkei Electronics 1983, 10(10), 173-194.

19. ECMAStandardizing Information and Communication System Standard ECMA-267, December 1997.

20. Saimi, T.; Mizuno, S.; Itoh, N. Amelioration of tracking signals by using slit-detection method. Proc. Conference of Japan Society of Applied Physics 1987, 34,29a-ZL-7, 743; Tokyo, March 1987.

21. Oudenhuysen, Ad.; Lee, Wai-Hon. Optical component inspection for data storage applications. Proc. SPIE Optical Mass Data Storage II 1986, 695, 206-214.

22. Tsunoda, Y. On-land composite pregrove method for high tract density recording. Proc. SPIE Optical Mass Data Storage I 1986, 695, 224-229.

23. Saito, A.; Maeda, T.; Tunoda, Y. Fast Accessible Optical Pick-up, O plus E; Shingijyutsu Communications: Japan, 1986, 76, 84-87.

24. Boivin, A. Théorie et Calcul des Figures de Diffractions; Press de l'Université Laval: Quebec, 1964; 118-122.

25. Maréchal, A.; Françon, M. Diffraction Structure des Images; Masson \& Cie: Paris, 1970; 105-112. 